\title{
Lessons Learned from Applying Varying Coefficient Model to Controlled Simulation Datasets
}

\author{
Xiaofeng Meng ${ }^{1}$, Christine Goulet ${ }^{1}$ \\ ${ }^{1}$ Southern California Earthquake Center, University of Southern California
}

\begin{abstract}
The development of site- and path-specific (i.e., non-ergodic) ground motion models (GMMs) can drastically improve the accuracy of probabilistic seismic hazard analyses (PSHA). The Varying Coefficient Model (VCM) is a novel technique for developing non-ergodic GMMs, which puts epistemic uncertainty into spatially varying coefficients. The coefficients at nearby locations are correlated by placing a Gaussian process prior on them. The correlation structure is determined by the data, and later used to predict coefficients and their epistemic uncertainties at new locations. It is important to carefully verify the technique before its results can be accepted by the engineering community. In this study, we used a series of simulation-based controlled ground motion datasets from CyberShake to test a modified VCM technique, which partitions the epistemic uncertainty into spatially varying source, site and path terms. Because the simulation parameters (inputs) are known, it is straightforward to verify what is recovered by the VCM from CyberShake simulation. We find that the site effects in CyberShake datasets can be reliably recovered by the VCM. However, the densely-located self-similar events in CyberShake datasets lead to large correlation lengths, which violates the isotropic assumption underlying the method and prevents the VCM from capturing the genuine source effects. For path effects, cell-specific attenuation approaches fail to recover the anelastic attenuation pattern of the 3D velocity model, most likely due to inappropriate assumption of point sources and straight-line wave propagation. Instead, a midpoint approach that only considers the aggregated path effects can better recover the strong attenuation within basins by fixing the correlation length of path effects. Lessons learned in this study not only provide important guidance for future applications of VCM to both simulation and empirical datasets, but also help further development of the technique, notably for the recovery of path effects.
\end{abstract}




\section{Introduction}

Probabilistic seismic hazard analysis (PSHA) is the main approach considered for engineering design of buildings and civil infrastructure. PSHA relies on two complementary models: an earthquake rupture forecast (ERF, which defines the expected rate and location of earthquakes of varying magnitudes in a region) and a ground motion model (GMM, which provides the expected ground motions conditioned on the ERF). The main product of PSHA is hazard curves relating the probability of exceedance of given ground motion levels. In the GMM, the total standard deviation of the residuals $\left(\sigma_{t o t}\right)$ between ground motion measurements and the GMM median predictions represents the variability of ground motions expected at a site. Larger variability leads to higher ground motions at any given annual frequency of exceedance in PSHA results, especially at the long return periods of engineering interest. Strasser et al., (2009) showed that $\sigma_{\text {tot }}$ did not decrease in the past four decades despite substantial increase in the number of strong-motion recordings and complexity of the GMMs. The ergodic assumption used in GMM development, which postulates that the aleatory variability of ground motions at one site over time equals the variability of ground motions over all sites in a short time interval (i.e., $\sigma_{t o t}$ ), is the primary cause for the persistent large variability. In reality a significant part of $\sigma_{t o t}$ comes from systematic variability spanning different source regions, sites and wave propagation paths. Yet, site response and path effects at a given location, for example, are expected to be similar under similar conditions, and we can consider them as repeatable or part of the epistemic uncertainty. Therefore, the most promising way to improve the accuracy of hazard curve calculations is through the removal of the ergodic assumption, that is, separating aleatory and epistemic variabilities.

Several approaches have been proposed to remove ergodicity in GMMs. One of the most commonly referred frameworks is developed by Al Atik et al., (2010). Under this framework, the total residuals from a GMM regression are partitioned into three epistemic components (repeatable source, path and site effects), as random effects (i.e., independent point estimates) in mixed-effects analysis, and two aleatory components (single-region between-event and within-event single-path residuals). For the past decade, this framework has been adopted for most non-ergodic GMM developments (Lin et al. 2011; Dawood and Rodriguez-Marek 2013; Villani and Abrahamson 2015; Stewart et al. 2017). An alternative approach, proposed by Wang and Jordan (2014), decomposes the total residuals into independent site, path, directivity and 
source-complexity effects using the average-based factorization technique. More recently, Landwehr et al., (2016) developed a non-ergodic GMM for California using a novel technique known as the Varying Coefficient Model (VCM). In that work, the GMM coefficients are allowed to vary spatially with locations, which is achieved by defining a Gaussian process prior over each coefficient. The correlation length and variability of each coefficient is determined by the data. The epistemic source, site, and path effects are put into the VCM coefficients, as opposed to being treated as independent point estimates as done in the Al Atik et al., (2010) framework. At locations with data, the location-specific GMMs have reduced epistemic uncertainty, while at locations without data, the GMMs resort back to the ergodic model with larger uncertainty. By capturing the different sources of variability within the coefficients themselves, the VCM approach is considered a statistically more viable approach to developing non-ergodic GMMs.

Since the VCM is a novel and promising technique, a Non-Ergodic Working Group (NEWG) was formed to advance the understanding of the approach, propose modifications, and perform preliminary applications on various datasets; this special issue captures the work completed by the NEWG in the last few years (Lavrentiadis 2021). Coordinated within the NEWG, our role is to perform verification and sensitivity studies of the VCM technique using simulated ground motion datasets. Ground motion simulation datasets present two major advantages over empirical datasets. First, simulations can be obtained for any source regions, sites, and paths, which can all be sampled many times. Hence, compared to the sparse empirical datasets, the source, site and path effects can be more accurately estimated. Second, since the source parameters of earthquakes, site conditions, and the underlying velocity model used in simulations are known, the overall accuracy of the VCM outputs can be directly verified against the inputs.

In this study, we use the Southern California Earthquake Center CyberShake ground motion simulation platform (hereafter CyberShake). CyberShake is, by definition, a nonergodic PSHA model; it incorporates an ERF with a full 3D wave propagation to perform physics-based PSHA by computing ground motions at chosen sites (Graves et al. 2010; Jordan et al. 2018). At each site, seismograms are calculated using seismic reciprocity for all ruptures within $200 \mathrm{~km}$ from the Uniform California Earthquake Rupture Forecast, Version 2 (UCERF2) model (Field et al. 2009). The ruptures themselves are generated using a kinematic source model (Graves and 
Pitarka 2010, 2015) and the wave propagation properties are based on large regional 3D community velocity models (CVMs). CyberShake includes up to 415 variations (i.e., rupture scenario) for each rupture (with the same magnitude and rupture plane) defined in UCERF2. Each rupture variation is essentially an unique event in the CyberShake datasets. RotD50 pseudo-spectral acceleration (PSA) at periods of 2, 3, 5 and 10s are calculated from each simulated seismogram.

We adopt a modified VCM technique largely based on Landwehr et al., (2016). Instead of directly solving for a non-ergodic GMM with spatially-varying coefficients, we first obtain an ergodic GMM through linear regression. Following the regression, we apply the VCM to the total residuals and compute the spatially varying source, site and path effects. The advantage of the modification is two-fold (1) it significantly reduces the computational cost by decreasing the number of spatially varying components from a dozen to three, which is a primary concern for very large simulation datasets; and (2) the outputs have a similar decomposition to those from $\mathrm{Al}$ Atik et al., (2010). Thus the results can be easily understood by the earthquake engineering community and compared to previous studies (Villani and Abrahamson 2015; Meng and Goulet 2021).

The modified VCM is applied to a CyberShake study in southern California (hereafter CS15.4). CS15.4 includes 336 sites, 360,472 events and 97,214,974 seismograms (Figure 1a), from which we select subsets of simulation data. It would be impractical to apply the VCM to the entire CS15.4 dataset, as it requires an extraordinary amount of memory and computation time. Moreover, the main purpose of this work is to verify the VCM and test its sensitivity with a controlled dataset, and not to develop a non-ergodic GMM based on CyberShake data. Therefore, we first randomly generate four reduced CS15.4 datasets of different sizes. Then, VCM is applied to the four datasets, and the outputs are compared among themselves to check the sensitivity on dataset sizes. Next, we identify results that are consistent with the input components, which can be used to better constrain future PSHA applications. When discrepancies emerge between inputs and outputs, we explore their causes by conducting more tests on controlled datasets and propose potential solutions for future VCM applications. 


\section{Methodology}

We define a simple function form to develop the ergodic GMM, which includes magnitude $(M)$ scaling, geometric spreading, magnitude-distance interaction and linear site response:

$$
f_{\text {erg }}=c_{0}+c_{1} M+c_{2} M^{2}+\left(c_{3}+c_{4} M\right) \cdot \log \left(\sqrt{R_{r u p}^{2}+h^{2}}\right)+f\left(V_{S 30}\right)
$$

where $h$ is set to $4.5 \mathrm{~km}$ and $c_{i}$ are coefficients determined through regression; $R_{r u p}$ is the closest distance between the site and the rupture plane; $f\left(V_{S 30}\right)$ is the linear site response term based on the slowness-averaged shear wave velocity in the top $30 \mathrm{~m}$ of the velocity model $\left(V_{S 30}\right)$ :

$$
\begin{array}{cc}
f\left(V_{S 30}\right)=c_{5} \cdot \log \left(\min \left(V_{S 30}, 800\right) / 800\right) & V_{S 30} \leq 1500 \mathrm{~m} / \mathrm{s} \\
f\left(V_{S 30}\right)=c_{6} \cdot \log \left(V_{S 30} / 1500\right) & V_{S 30}>1500 \mathrm{~m} / \mathrm{s}
\end{array}
$$

The separation point of $f\left(V_{S 30}\right)$ is set as $1500 \mathrm{~m} / \mathrm{s}$ as it is the upper limit of the $V_{S 30}$ values from the NGA-West2 dataset (Ancheta et al. 2014).

The total residuals are decomposed into three spatially varying components (source, site, path effects) and the remaining aleatory residuals:

$$
y_{e s}=f_{e r g}+\delta L 2 L\left(\overrightarrow{x_{e}}\right)+\delta S 2 S\left(\overrightarrow{x_{s}}\right)+\delta P 2 P\left(\vec{x}_{e^{\prime}} \overrightarrow{x_{s}}\right)+\delta B_{e}^{0}+\delta W S_{e, s}^{0}
$$

where $\delta L 2 L\left(\overrightarrow{x_{e}}\right)$ are the source effects that spatially vary with event locations $\vec{x}_{e}, \delta S 2 S\left(\overrightarrow{x_{s}}\right)$ are the site effects that spatially vary with site locations $\vec{x}_{s}, \delta P 2 P\left(\vec{x}_{e}, \overrightarrow{x_{s}}\right)$ are the spatially varying path effects, $\delta B_{e}^{0}$ and $\delta W S_{e, s}^{0}$ are the remaining residuals after all spatial correlations are considered. For this paper, we experiment with three modeling approaches for $\delta P 2 P\left(\vec{x}_{e}, \overrightarrow{x_{s}}\right)$, all of them being simplifications relative to the complex source-site travel paths. The first approach is the 2 dimensional (2D) cell-specific attenuation (hereafter 2D cell approach), similar to the one introduced by (Dawood and Rodriguez-Marek 2013). The entire study region is divided into $2 \mathrm{D}$ cells of $0.25^{\circ}$ by $0.25^{\circ}$. The path effects are the sum of anelastic attenuation from all cells along a horizontal straight line from the closest point to the site:

$$
\delta P 2 P(\vec{x}, \vec{x})=\sum_{i} \delta_{i} \cdot \Delta R_{i}
$$


where $\delta_{i}$ is the anelastic attenuation per kilometer of travel in the $i^{\text {th }}$ cell. In theory, $\delta_{i}$ represents the combined effects of anelastic attenuation and scattering, but we refer to it as the anelastic attenuation term hereafter for simplicity. $\delta_{i}$ is computed as an independent random effect during regression (i.e., no spatial correlation). $\Delta R_{i}$ is the approximate travel distance within the $i^{\text {th }}$ cell. The second approach is the 3 dimensional (3D) cell-specific attenuation (hereafter 3D cell approach). The entire subsurface is divided vertically into $1 \mathrm{~km}$ layers. In the top $10 \mathrm{~km}$, each layer is further divided into cells of $0.25^{\circ}$ by $0.25^{\circ}$. Below $10 \mathrm{~km}$, each layer is no longer divided into small cells, because there is very little heterogeneity at larger depths in the $3 \mathrm{D}$ velocity structure used for the simulations. This implementation provides the additional advantage of significantly reducing the computational demands. The wave propagation is assumed as a straight line from the hypocenter to the site. The third approach only considers the aggregate path effects along the entire path. Similar to the path term described in Al Atik et al., (2010), each path between a source region and a site is considered unique. We use the midpoint $t_{p}$ between the site and the closest point of the causative fault to represent the approximate location of a path:

$$
\delta P 2 P\left(\overrightarrow{x_{e}}, \overrightarrow{x_{s}}\right)=\beta_{1}\left(\vec{x}_{m p}\right) \times R_{\text {rup }}
$$

where $\beta_{1}\left(x_{m p}\right)$ is anelastic attenuation that spatially varies with midpoint locations $\vec{x}_{m p}$.

Hereafter this approach will be referred to as the midpoint approach.

The next step is to determine the amount of correlation in $\delta L 2 L\left(\vec{x}_{e}\right), \delta S 2 S\left(\vec{x}_{s}\right)$ and $\beta_{1}\left(x_{m p}\right)$. This is achieved by imposing a Gaussian process (Rasmussen et al. 2006) prior on the three terms. We use the Matern kernel to constrain the spatial correlation between any two data points:

$$
C_{v}(r)=\omega^{2} \frac{2^{1-v}}{\Gamma(v)}\left(\sqrt{8 v} \frac{r}{l}\right)^{v} K_{v}\left(\sqrt{8 v} \frac{r}{l}\right)
$$

where $\Gamma(v)$ is the gamma function, $K_{v}$ is the modified Bessel function of the second kind, $r$ is the distance in degree between two data points; $v$ is the smoothness parameter, which is fixed at 1 in this study; $l$ and $\omega$ are hyperparameters that represent the correlation length and standard deviation, respectively, which are determined by the data. The Markov-chain Monte Carlo (MCMC) approach is often used to solve the hyperparameters of a Gaussian process, but it tends to be very computationally expensive because it computes the joint posterior distribution of the 
hyperparameters. The Integrated Nested Laplace Approximations (INLA) method was recently developed as a fast alternative to the Bayesian inference method (Rue et al. 2009). Instead of computing the joint posterior distribution of the hyperparameters like in the MCMC approach, the INLA method computes deterministic approximations of the hyperparameters. INLA has great scalability with very large datasets, and can be easily implemented with the R-INLA package [www.r-inla.org]. In our test run with a sample CyberShake dataset of 200 earthquakes and 336 stations, R-INLA achieves up to $\sim 10$ times speed up compared to the MCMC approach and the differences of results are negligible. The fast computations from R-INLA were instrumental in our ability to run the several rounds of tests needed to develop this publication.

In R-INLA, the spatial correlation of data is estimated by Stochastic Partial Differentiation Equation (SPDE) (Lindgren et al. 2011), which uses a finite element method to represent a Gaussian process. First, a mesh consisting of triangles is created over the study region. The continuous spatial processes are then discretized onto the vertices of the triangles. If a data point is located within a triangle, three vertices are directly related to the data point weighted by their respective distance to it. If a data point is located on the edge of a triangle, only two vertices at two ends are related to it and weighted by distances. If a data point is located on one vertex of a triangle, the other two vertices are not related to it. The total weight of each data point is always 1. Next, the SPDE algorithm calculates the correlation structure among all the vertices of the mesh, which is then used to make predictions at any location. Smaller triangles lead to higher precision but require larger memory and longer computation times. After many trial runs with the reduced CyberShake datasets, we find the most satisfying triangle size for the balance of the precision and computational cost is $0.1^{\circ}$.

\section{Datasets}

The magnitude- $R_{r u p}$ and $V_{S 30}$ distributions of CS15.4 are shown in Figure $1 \mathrm{~b}$ and $1 \mathrm{c}$, respectively. To test the sensitivity of VCM to dataset size, we generate four reduced datasets by randomly selecting 100, 200, 600, and 1000 events in CS15.4 (Table 1 and Figure 2). While these are reduced datasets relative to the whole CS15.4 simulation set, the corresponding number of data is still quite large (Table 1); in comparison, the NGA-West2 database contains 21,336 records spanning 599 events. Since a single rupture in CyberShake includes up to hundreds of variations, we require that only one rupture variation per rupture be chosen so that the reduced 
datasets have the largest variability in source location and parameters. There are 336 sites in CS15.4, which are all included in the reduced datasets. The sites are concentrated within a box centered around Los Angeles, which covers the Los Angeles and Ventura sedimentary basins and their immediate vicinity; two additional test sites are located away from the box. The 3D velocity model used for the CS15.4 simulations is CVM-S4.26-M01, obtained from full three-dimensional waveform tomography (F3DT) (Lee et al. 2011; Small et al. 2017) and supplemented by borehole information for near-surface velocities (Ely et al. 2010). The shear-wave velocity $\left(V_{S}\right)$ has a floor value of $500 \mathrm{~m} / \mathrm{s}$. Outside the range of CVM-S4.26-M01, a generic 1D velocity model is used. We use RotD50 PSA at 5s as the ground motion measure, the same period used for the development and validation of the 3D velocity model (Lee et al. 2011; Lee and Chen 2016).

The effect of anelastic attenuation is usually specified with quality factor, $Q$. However, CVM-S4.26-M01 only provides the seismic velocities and density of the material, and we use an empirical relationship to invert $Q$ from the seismic velocity model (Taborda and Bielak 2013):

$$
Q_{S}=10.5-16 V_{S}+153 V_{S}^{2}-103 V_{S}^{3}+34.7 V_{S}^{4}-5.29 V_{S}^{5}+0.31 V_{S}^{6}
$$

The $Q_{S}$ maps are then used as the reference for the input attenuation (Figure 3). Near the surface, there are two regions with much stronger attenuation than the rest of southern California. The first region includes the Ventura and Los Angeles basins, as well as their immediate vicinity; the second one is the Imperial Valley to the south. The areas of the two regions quickly decrease with depth such that below $5 \mathrm{~km}$, the attenuation within the two regions becomes very similar to most of the study region, leaving the Great Valley (to the north) as the only region with stronger attenuation.

Table 1

\begin{tabular}{|c|c|c|}
\hline Dataset name & Number of events & Number of records \\
\hline CS15.4-100 & 100 & 21,269 \\
\hline CS15.4-200 & 200 & 56,252 \\
\hline CS15.4-600 & 600 & 159,659 \\
\hline CS15.4-1000 & 1000 & 265,252 \\
\hline
\end{tabular}




\section{Results}

There is no apparent trend in the total residuals relative to any of the predictor variables for the four datasets (Figure S1-S3), suggesting no bias in the linear regression results. Figure 4 and 5 show the maps of $\delta L 2 L\left(\overrightarrow{x_{e}}\right)$ and the epistemic predictive uncertainty $\omega_{L 2 L}\left(\overrightarrow{x_{e}}\right)$ for the four datasets, respectively. For CS15.4-100, the variations of $\delta L 2 L\left(\overrightarrow{x_{e}}\right)$ are constrained to the event locations (Figure 4a). At locations without events, $\delta L 2 L\left(\overrightarrow{x_{e}}\right)$ returns to the median value (i.e., zero) and the predictive uncertainty $\omega_{L 2 L}\left(\overrightarrow{x_{e}}\right)$ increases significantly, as intended (Figure $4 \mathrm{a}$ and 5a). For the three larger datasets, the correlation lengths $l$ of $\delta L 2 L\left(\overrightarrow{x_{e}}\right)$ increase significantly and as a result, the variations of $\delta L 2 L\left(\overrightarrow{x_{e}}\right)$ extend to large areas without events (Figure $4 \mathrm{~b}-\mathrm{d}$ ). Moreover, there is no longer a clear distinction in $\omega_{L 2 L}\left(\overrightarrow{x_{e}}\right)$ between locations with and without data for the three larger datasets (Figure 5b-d). In other words, the VCM fails to capture the genuine source effects when the number of events in CyberShake datasets increases.

The results of $\delta S 2 S\left(\vec{x}_{S}\right)$ and its predictive uncertainty $\omega_{S 2 S}\left(\vec{x}_{S}\right)$ are almost identical across four datasets (Figure 6 and 7). Within the Ventura and Los Angeles basins, the sites have the strongest site responses. In comparison, the hard rock sites at the San Gabriel Mountains have much weaker site responses. The site responses return to zero outside of the site coverage, where they show increased epistemic uncertainty. The few test sites located outside of the box show the same trends. The consistent pattern suggests that the genuine site effects of CyberShake datasets are accurately recovered by the VCM technique.

For the 2D cell approach, the variations of $\delta_{i}$ are constrained at locations with data (Figure 8), and the uncertainty of $\delta_{i}$ increases at locations without data (Figure 9). However, the approach fails to capture the strong attenuations within the Ventura and Los Angeles basins observed in the $Q_{S}$ maps (Figures 3 and 8). In all four datasets, cells within the basins and their immediate vicinity (hereafter inner cells) have $\delta_{i}$ close to zero (Figure 8), which represent the median attenuation of all the cells sampled by seismic waves. Outside the basins (hereafter outer cells), the $\delta_{i}$ patterns are dependent on the datasets and have no consistency with the $Q_{S}$ maps at any depth. 
The $\delta_{i}$ results obtained by the 3D cell approach show slight improvement over the 2D cell approach. At shallow depths $(0-3 \mathrm{~km})$, the outer cells have consistent weak attenuation, especially for the three larger datasets, which agree with the $Q_{S}$ maps. However, $\delta_{i}$ of the inner cells at shallow depths are still close to zero for four datasets, instead of the expected strongest attenuations (Figure 10). The number of inner cells with close-to-zero $\delta_{i}$ quickly decreases with depth. Below $5 \mathrm{~km}$, there is no longer a distinct area with close-to-zero $\delta_{i}$ for all the datasets. Cells with weak and strong attenuation are located randomly, with very little agreement with the $Q_{S}$ maps at the corresponding depth.

In comparison with the $2 \mathrm{D}$ and $3 \mathrm{D}$ cell approaches, the midpoint approach enables to recover strong attenuations within the basins for the four datasets (Figure 11), and the predictive uncertainties $\omega_{P 2 P}\left(x_{m p}\right)$ increase at locations without data (Figure 12). However, similar to $\delta L 2 L\left(\overrightarrow{x_{e}}\right)$, the variations of $\beta_{1}\left(x_{m p}\right)$ extend to large areas without data due to the very large correlation lengths (Figure 11). Moreover, the correlation lengths are evidently different among the four datasets, which result in significantly different patterns outside the basins. Therefore, although the midpoint approach obtains results that are more consistent with the input velocity models, there are still remaining issues on the stability of the approach.

\section{Discussion}

In this section, we discuss the results for each of the spatially variable results in more detail. One point of note is that in addition to these interpretations, it is possible that the approach we used, in which we first perform a linear regression and then apply the VCM approach to the residuals could introduce a bias. However, because the CS15.4 is complete (all events have estimates at all sites and vice-versa), we do not believe this to be a source of concern.

\subsection{Source effects $\delta L 2 L\left(\overrightarrow{x_{e}}\right)$}

The very large correlation lengths of $\delta L 2 L\left(\overrightarrow{x_{e}}\right)$ are likely caused by two unique properties of the CyberShake datasets. First, the majority of events are self-similar, that is, they follow the same magnitude-rupture area relationship (i.e., same stress drop) (Somerville et al. 1999). Second, the majority of events in CyberShake datasets are associated with principal faults in California. When the number of events becomes large, they occur densely along those faults and 
form large linear features (Figure 2). The self-similar events along large linear features thus lead to large correlation lengths of $\delta L 2 L(\vec{x})$. In R-INLA, the Gaussian Process is assumed isotropic, that is, that the process only depends on the distances between two data points, but not the azimuth. As a result, when predicting at new locations, the large correlation length is projected onto all azimuths. To test this hypothesis, we randomly selected another 600 events in CS15.4 (hereafter, CS15.4-600b) under two restrictions: 1) the minimum distance between any two events is $10 \mathrm{~km}$; 2) the stress drops are evenly distributed between 0 and $3 \mathrm{MPa}$ (Figure 13a). After applying VCM to CS15.4-600b, we find that the correlation length of $\delta L 2 L\left(\overrightarrow{x_{e}}\right)$ becomes much smaller (Figure 13b). The variations of $\delta L 2 L\left(\vec{x}_{e}\right)$ are constrained at event locations and correlate well with stress drops (Figure 13b). $\omega_{L 2 L}\left(\overrightarrow{x_{e}}\right)$ also increases at locations without data as intended (Figure 13c). This test confirms that large correlation lengths of $\delta L 2 L\left(\overrightarrow{x_{e}}\right)$ for CyberShake datasets are indeed caused by the dense linear distribution and uniform stress drop of events. Thus, for future application of VCM on CyberShake datasets, it is recommended to fix the correlation length at a very small value so that the source effects are constrained at event locations. This is essentially the same approach that computes event terms as point estimate random effects, as summarized by Al Atik et al., (2010).

In typical empirical datasets, earthquakes tend to be much more sparse and have a wide range of stress drops. Therefore, it is unlikely to run into the same problem of large correlation lengths. For example, the source effects obtained by VCM for California and France datasets do not exhibit such behaviors (Lavrentiadis et al. 2021; Sung et al. 2021). However, if an empirical dataset exclusively consists of one earthquake sequence or swarm, in which all the events have similar stress drop and are located densely along one fault, the VCM will likely yield large correlation lengths of $\delta L 2 L(\vec{x})$ and unreasonable predictions at locations without data.

\subsection{Site effects $\delta S 2 S\left(\overrightarrow{x_{s}}\right)$}

The large site responses observed within the Ventura and Los Angeles basins agree with the strong basin amplification observed from empirical data (e.g. Wald and Graves 1998). VCM does an excellent job capturing the basin effects from the CyberShake datasets. Provided that the 
CyberShake site effects are validated, they could be used directly to complement empirical GMMs and improve seismic hazard analysis within the site coverage. Based on the results of $\delta L 2 L\left(\vec{x}_{e}\right)$, it is reasonable to assume that, if most of the sites are in a dense linear array, the isotropic assumption of the Gaussian Process will likely yield a large correlation length of $\delta S 2 S\left(\vec{x}_{S}\right)$ as well. Hence, for future non-ergodic GMM development, one should take extra caution when dense linear arrays are involved.

\subsection{Path effects $\delta P 2 P\left(\vec{x}, \overrightarrow{x_{s}}\right)$}

Both the 2D and 3D cell approaches fail to capture the strongest attenuation at shallow depths within the Ventura and Los Angeles basins for any reduced dataset. Considering that almost all the paths go through the inner cells, the near-zero $\delta_{i}$ might be due to averaging of path effects from a wide azimuth range. For the outer cells, the entire path effects might be inferred from a small sample of paths with similar azimuth. To test this hypothesis, we perform tests on two controlled datasets, which were selected for the 2D and 3D cell approaches respectively. For both controlled datasets, the locations of events and sites are based on CS15.4-600. In this way, we reproduce the same paths in CS15.4-600. All components of the total residuals $(\delta L 2 L(\vec{x})$, $\delta S 2 S\left(\vec{x}_{s}\right), \delta P 2 P\left(\vec{x}_{e}, \vec{x}_{s}\right), \delta B_{e}^{0}$ and $\left.\delta W S_{e, s}^{0}\right)$ of the controlled datasets are generated randomly. In both controlled datasets, $\delta L 2 L\left(\vec{x}_{e}\right)$ and $\delta S 2 S\left(\vec{x}_{s}\right)$ are randomly drawn from normal distributions with standard deviation of 0.4 (i.e., $N\left(0,0.4^{2}\right)$ ), while $\delta B_{e}^{0}$ and $\delta W S_{e, s}^{0}$ are randomly drawn from $N\left(0,0.2^{2}\right)$. For the $2 \mathrm{D}$ approach, the study region is divided into 20 patches, each with a distinct attenuation rate $\delta_{i}$, which is randomly drawn from $N\left(0,0.001^{2}\right)$ (Figure 14a). $\delta P 2 P\left(\vec{x}_{e}, \vec{x}_{s}\right)$ is computed as the sum of attenuation of all cells from the closest point to the site (equation 6). For the $3 \mathrm{D}$ approach, $\delta P 2 P\left(\overrightarrow{x_{e}}, \overrightarrow{x_{s}}\right)$ is computed from $3 \mathrm{D}$ cells. In the top $10 \mathrm{~km}$, each layer is divided into 20 patches, with each a distinct attenuation rate $\delta_{i}$, which is also drawn from $N(0$, $0.001^{2}$ ) (Figure 15). Below $10 \mathrm{~km}$, the entire layer is one single patch, whose $\delta_{i}$ is drawn from the same normal distribution. The path effects are calculated as the cumulative attenuation along a straight line from the hypocenter to the site (equation 6). After applying the VCM to both 
controlled datasets, we find that the attenuation patterns can be accurately recovered in both cases (Figure 14, 15). For most cells, the differences between the output and input $\delta_{i}$ are very close to zero. The only non-zero differences occur at a few cells on the edge, which are only sampled by a few paths. This test disapproves of the hypothesis that the failure of the cell approaches is caused by the averaging of the large number of paths sampled by the inner cells.

To study the sensitivity of the cell approaches, we perform another test with the 3D controlled dataset. After computing the input $\delta P 2 P\left(\overrightarrow{x_{e}}, \overrightarrow{x_{s}}\right)$ from the hypocenter to site, we randomly shift the hypocenters by a small distance on the rupture plane $N\left(0, \sigma_{1}^{2}\right)$. Now there is a subtle difference between the path used in computing the input path effects and the path used for regression in the VCM. Even with a very small shift $\left(\sigma_{l}=1 \mathrm{~km}\right)$, significant deviations between the output and input $\delta$ are observed (Figure 16). As a result, it can be concluded that the cell approaches are highly sensitive to the assumption of seismic wave propagation paths. As mentioned in section 3, all the events in CS15.4 are above magnitude 6. The rupture plane of a magnitude 6 earthquake typically has a length of $30 \mathrm{~km}$ and a width of $12 \mathrm{~km}$. For such large rupture planes, the point source assumption used in the VCM is no longer appropriate. Moreover, there are strong refraction and reflection at interfaces where seismic wave velocities change significantly, as well as large surface waves in certain cases. This confirms that the simplification of considering seismic waves as travelling along a straight line from a point to a site is not appropriate, which is likely the primary cause for the failure of the cell approaches. In the near future, we will continue to investigate the more accurate representation of wave propagation paths in CyberShake simulations, so that the genuine anelastic attenuation can be captured.

Unlike the cell approaches, the midpoint approach only considers the aggregated attenuations from one event to one site, without any assumption of wave propagation path. As a result, the strongest attenuations within the basins are recovered. On the other hand, the midpoint approach also smoothes out small scale features in attenuation patterns by aggregating them, which results in large correlation lengths of $\delta P 2 P\left(\overrightarrow{x_{e}}, \overrightarrow{x_{s}}\right)$ (Figure 9). Since R-INLA does not allow specifying the lower- or upper-bound of the correlation length, we try to find alternative ways to constrain correlation length of $\delta P 2 P\left(\vec{x}_{e}, \vec{x}\right)$ during VCM regression. One way to quantify the spatial correlation of path effects is to apply the semivariogram model to path terms computed from mixed-effects regression (Walling and Abrahamson 2012). The range of values 
in the best-fitting semivariogram function denotes the lag distance beyond which path terms are no longer correlated. We first compute the path terms with the mixed-effects regression for CS15.4-1000 and perform a regression on the semivariogram function with a spherical model (Figure 17a). The best-fitting range value, 1.209, is used as the fixed correlation length of

$\delta P 2 P\left(\vec{x}_{e}, \overrightarrow{x_{s}}\right)$ in VCM regression. The updated pattern still captures the strongest attenuation within the basins and much weaker attenuation outside the basins (Figure 17b). More importantly, the weak attenuations are constrained at locations with data. This approach is promising for broader application but would require additional investigation using recorded data.

\subsection{Future work}

In this work, the spatial correlation of source and site effects are put into decomposed residual components from an ergodic GMM, while Landwehr et al., (2016) have spatially correlated GMM coefficients. The advantage of having spatially correlated GMM coefficients is that it is a more flexible framework to account for spatially correlated effects, which may better capture effects that are not included in source, site and path effects (e.g. directivity). On the other hand, the Landwehr et al., (2016) approach is more computationally expensive. Moreover, it did not take genuine path effects into account as the coefficients that account for distance scaling only considers an average distance attenuation for each event in all directions. Therefore, it would be important to systematically compare the results of different components between the approaches used in Landwehr et al., (2016) and in this paper in future work.

Another crucial finding from this work is that the path effects are very sensitive to the assumption of wave travel path. As a result, it is very challenging to compute path effects from large earthquakes from both empirical and simulation datasets, as their rupture planes may extend to several hundred kilometers and one single path assumption is not appropriate. Although the rupture planes of large earthquakes could be very large, they also often have patches of elevated slips that are responsible for the majority of seismic energy radiation (i.e. subevents). However, these issues can not be investigated with empirical datasets due to the paucity of large repeated events in a given region. Therefore, one possible solution is to conduct future ground motion simulations that include large and small earthquakes along the same rupture planes and study how path effects from the large earthquake relate to combinations of path effects from many sub-events or smaller earthquakes along the same rupture plane. Once a 
general relationship is established, we could approximate the path effects of future large earthquakes from combining path effects from smaller events (either empirical or simulated) on the same rupture plane.

\section{Conclusions}

In this study, we apply a novel technique, the VCM, to a CyberShake simulation dataset. By definition, CyberShake is a non-ergodic model, and the various inputs used for the simulations are known, making it an ideal testbed for better understanding and testing the VCM capabilities. We find that the obtained site effects are consistent with the simulation datasets. Furthermore, they are consistent in trends with empirical datasets, suggesting they could be extracted using the VCM and used to improve future non-ergodic GMMs for common site coverage. The VCM fails to capture the simulated source effects because the dense linear distribution of self-similar events in the CyberShake dataset violates the isotropic assumption required by the Gaussian Process. In this case, one may compute the point-estimate of the event term instead. For the path effects, both the 2D and 3D cell approaches fail to capture the anelastic attenuation from the 3D velocity model. Because CyberShake only has moderate to large magnitude events, the large rupture planes and surface wave generation lead to rather complicated wave propagation effects. It is inappropriate to assume that seismic waves propagate along a straight line between two points. A more sophisticated representation of wave propagation is necessary to recover the anelastic attenuation pattern. This finite-fault effect was one of the motivations for the development of large-scale simulations in the first place. However, the midpoint approach tends to better recover the attenuation patterns, especially when fixed correlation length is carefully selected, as it does not make any assumption on the wave propagation path.

This study provides important guidance for future applications of VCM to CyberShake datasets and other simulation datasets. Moreover, for empirical datasets that include linear features (e.g., earthquakes along one fault and linear seismic array), it is also crucial to consider the possible large correlation lengths. Last but not least, lessons learnt here will help further development of the technique to capture the genuine path effects for non-ergodic modelling development, both from simulations and as part of GMMs. 


\section{Acknowledgment}

This work was supported by the Southern California Earthquake Center (SCEC), SCEC Contribution \#xxxx. SCEC is funded by the National Science Foundation (NSF) and U.S. Geological Survey (USGS) through cooperative agreements with the University of Southern California (USC). Additional funding for this work was provided by the Pacific Gas \& Electric Company (PG\&E). This work used allocations from the Extreme Science and Engineering Discovery Environment (XSEDE) program, which is supported by National Science Foundation grant number ACI-1548562. Specifically, it used the Bridges and Bridges-2 system, which is supported by NSF award number ACI-1445606, at the Pittsburgh Supercomputing Center (PSC).We would like to thank Nicolas Kuehn, Chih-Hsuan Sung, Grigorios Lavrentiadis and Kevin Milner for comments and suggestions on this article. We also thank Scott Callaghan for his help with the CyberShake data.

\section{Declarations}

Conflict of interest The authors have no conflicts of interest to declare that are relevant to the content of this article.

\section{References}

Al Atik L, Abrahamson NA, Bommer JJ, et al (2010) The Variability of Ground-Motion Prediction Models and Its Components. Seismol Res Lett 81:794-801

Ancheta TD, Darragh RB, Stewart JP, et al (2014) NGA-West 2 Database. Earthquake Spectra 30:989-1005

Dawood HM, Rodriguez-Marek A (2013) A Method for including path effects in ground-motion prediction equations: An example using the Mw 9.0 Tohoku earthquake aftershocks. Bull Seismol Soc Am 103:1360-1372

Ely GP, Jordan TH, Small P, Maechling PJ (2010) A Vs30-derived near-surface seismic velocity model. In: Abstract S51A-1907, Fall Meeting. AGU San Francisco, CA

Field EH, Dawson TE, Felzer KR, et al (2009) Uniform California earthquake rupture forecast, version 2 (UCERF 2). Bull Seismol Soc Am 99:2053-2107

Graves R, Jordan TH, Callaghan S, et al (2010) CyberShake: A Physics-Based Seismic Hazard Model for 
Southern California. Pure Appl Geophys 168:367-381

Graves R, Pitarka A (2015) Refinements to the Graves and Pitarka (2010) Broadband Ground-Motion Simulation Method. Seismological Research Letters 86:75-80

Graves RW, Pitarka A (2010) Broadband ground-motion simulation using a hybrid approach. Bull Seismol Soc Am 100:2095-2123

Jordan TH, Callaghan S, Graves RW, et al (2018) Cybershake Models of Seismic Hazards in Southern and Central California. In: Proc. 11th Natl. Conf. Earthq. Eng. Earthq. Eng. Res. Institute, Los Angeles, CA. Earthq. Eng. Res. Institute

Landwehr N, Kuehn NM, Scheffer T, Abrahamson NA (2016) A nonergodic ground-motion model for California with spatially varying coefficients. Bull Seismol Soc Am 106:2574-2583

Lavrentiadis G (2021) Introduction to Development of Non-Ergodic Ground-Motion Models. Bull Earthquake Eng in review:

Lavrentiadis G, Abrahamson NA, Kuehn NM (2021) A Non-ergodic Effective Amplitude Ground-Motion Model for California. Bull Earthquake Eng in review:

Lee E, Chen P (2016) Improved Basin Structures in Southern California Obtained Through Full-3D Seismic Waveform Tomography (F3DT). Seismol Res Lett 87:874-881

Lee E-J, Chen P, Jordan TH, Wang L (2011) Rapid full-wave centroid moment tensor (CMT) inversion in a three-dimensional earth structure model for earthquakes in Southern California. Geophys J Int $186: 311-330$

Lindgren F, Rue H, Lindström J (2011) An explicit link between Gaussian fields and Gaussian Markov random fields: the stochastic partial differential equation approach. J R Stat Soc Series B Stat Methodol 73:423-498

Lin PS, Chiou BS-J, Abrahamson NA, et al (2011) Repeatable source, site, and path effects on the standard deviation for empirical ground-motion prediction models. Bull Seismol Soc Am 101:2281-2295

Meng X, Goulet CA (2021) Fully Nonergodic Ground Motion Models in California Using CyberShake and NGA-West2 Datasets. Bull Seismol Soc Am in prep:

Rasmussen CE, Williams CKI, Bach F (2006) Gaussian Processes for Machine Learning. MIT Press

Rue H, Martino S, Chopin N (2009) Approximate Bayesian inference for latent Gaussian models by using integrated nested Laplace approximations. J R Stat Soc Series B Stat Methodol 71:319-392

Small P, Gill D, Maechling PJ, et al (2017) The SCEC Unified Community Velocity Model Software Framework. Seismol Res Lett 88:1539-1552

Somerville P, Irikura K, Graves R, et al (1999) Characterizing Crustal Earthquake Slip Models for the Prediction of Strong Ground Motion. Seismol Res Lett 70:59-80

Stewart JP, Afshari K, Goulet CA (2017) Non-Ergodic Site Response in Seismic Hazard Analysis. Earthquake Spectra 33:1385-1414 
Strasser FO, Abrahamson NA, Bommer JJ (2009) Sigma: Issues, Insights, and Challenges. Seismol Res Lett 80:40-56

Sung C-H, Abrahamson NA, Kuehn NM, et al (2021) A non-ergodic ground-motion model of Fourier amplitude spectra for France. Bull Earthquake Eng in review:

Taborda R, Bielak J (2013) Ground-Motion Simulation and Validation of the 2008 Chino Hills , California , Earthquake. Bull Seismol Soc Am 103:131-156

Villani M, Abrahamson NA (2015) Repeatable Site and Path Effects on the Ground-Motion Sigma Based on Empirical Data from Southern California and Simulated Waveforms from the CyberShake Platform. Bulletin of the Seismological Society of America 105:2681-2695

Wald DJ, Graves RW (1998) The seismic response of the Los Angeles basin, California. Bull Seismol Soc Am 88:337-356

Walling MA, Abrahamson NA (2012) Non-ergodic probabilistic seismic hazard analyses. In: Proc. 15th World Conf. Earthquake Eng., Lisbon, Portugal, Paper

Wang F, Jordan TH (2014) Comparison of Probabilistic Seismic-Hazard Models Using Averaging-Based Factorization. Bull Seismol Soc Am 104:1230-1257 

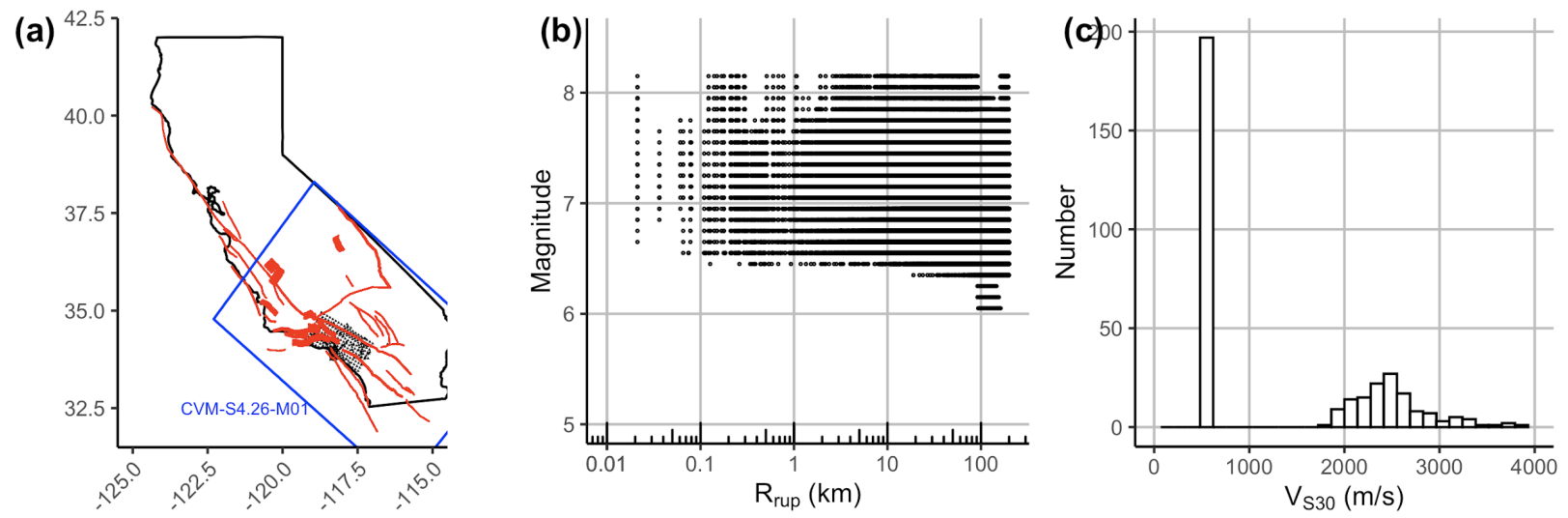

Figure 1. (a) Map of CS15.4; red dots and black triangles denote events' hypocenters and station locations (sites), respectively; the blue box outlines the extension of the $3 \mathrm{D}$ velocity model. (b) Magnitude- $R_{\text {rup }}$ distribution of events in CS15.4. (c) $V_{S 30}$ distribution of sites in CS15.4.

(a)

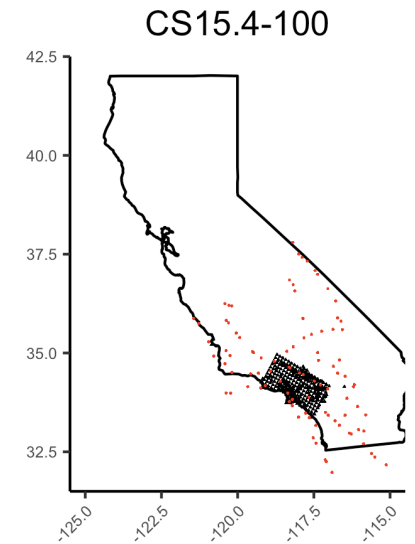

(c)

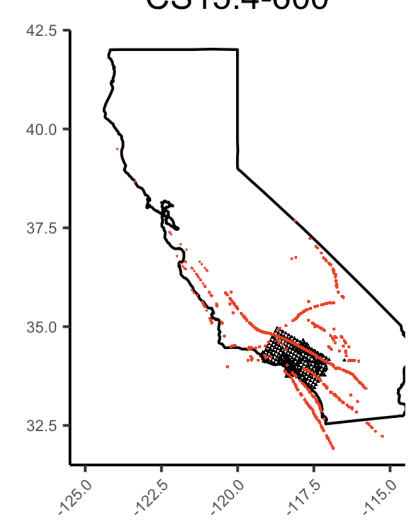

(b)

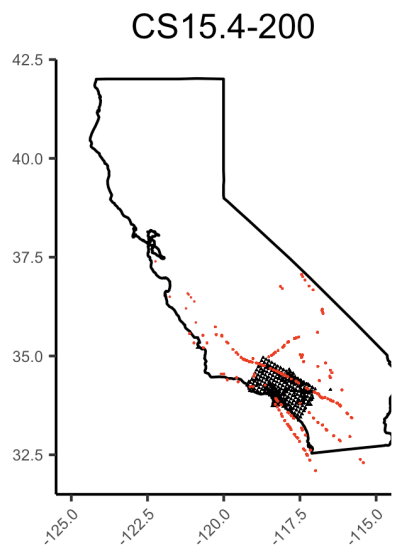

(d)
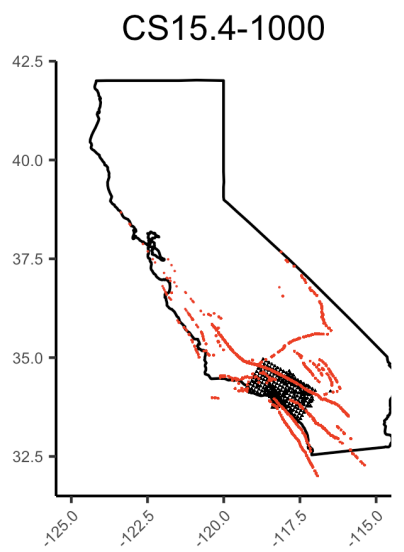

Figure 2. Map of four reduced CS15.4 datasets: red dots and black triangles denote events' hypocenters and station locations, respectively. Most of the sites are concentrated in the Los Angeles area, but a few are scattered farther away. 

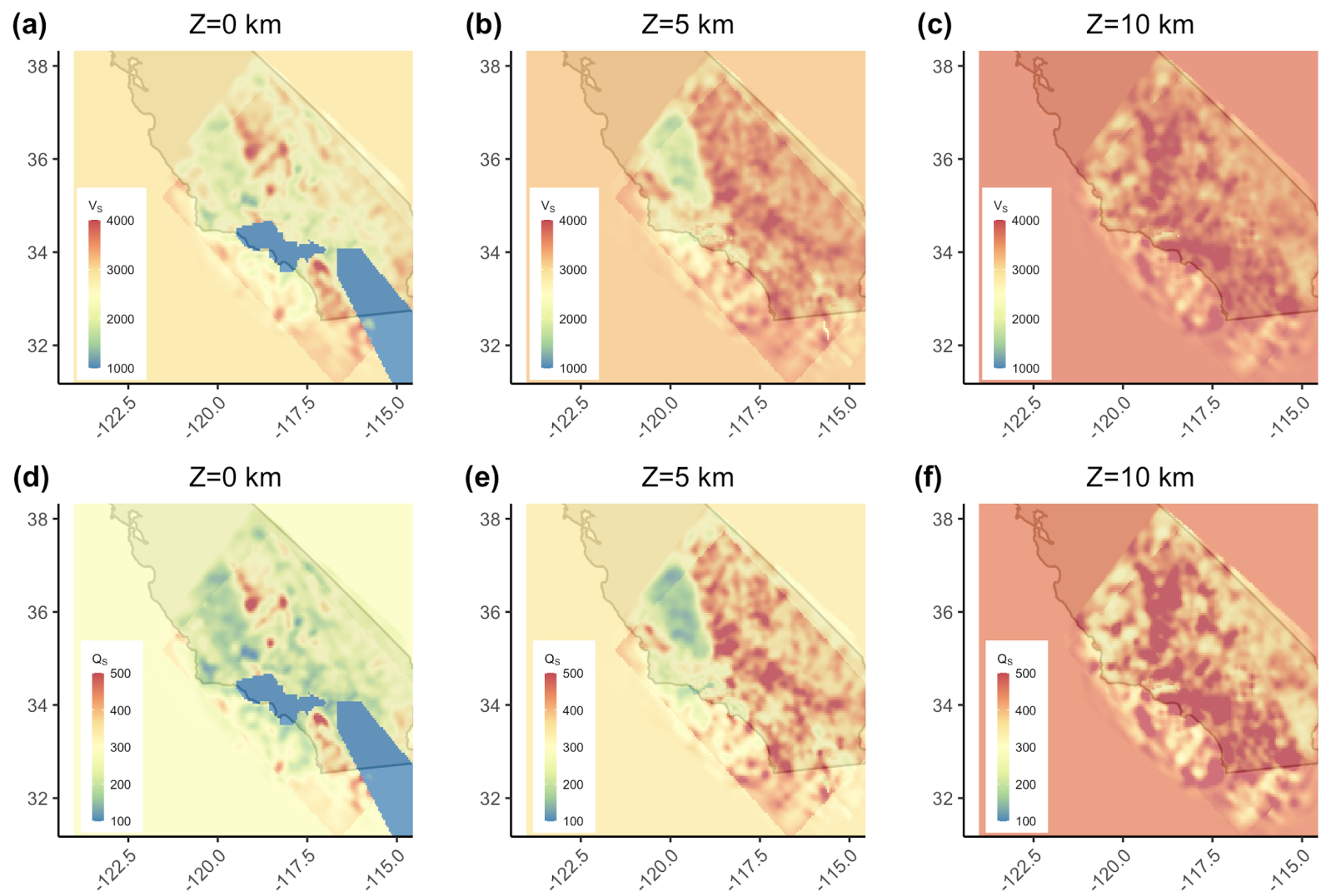

Figure 3. (a-c) Map of $V_{s}$ from CVM-S4.26-M01 at depth Z=0, 5, $10 \mathrm{~km}$. (d-f) Map of $Q_{s}$ at the same depths. 

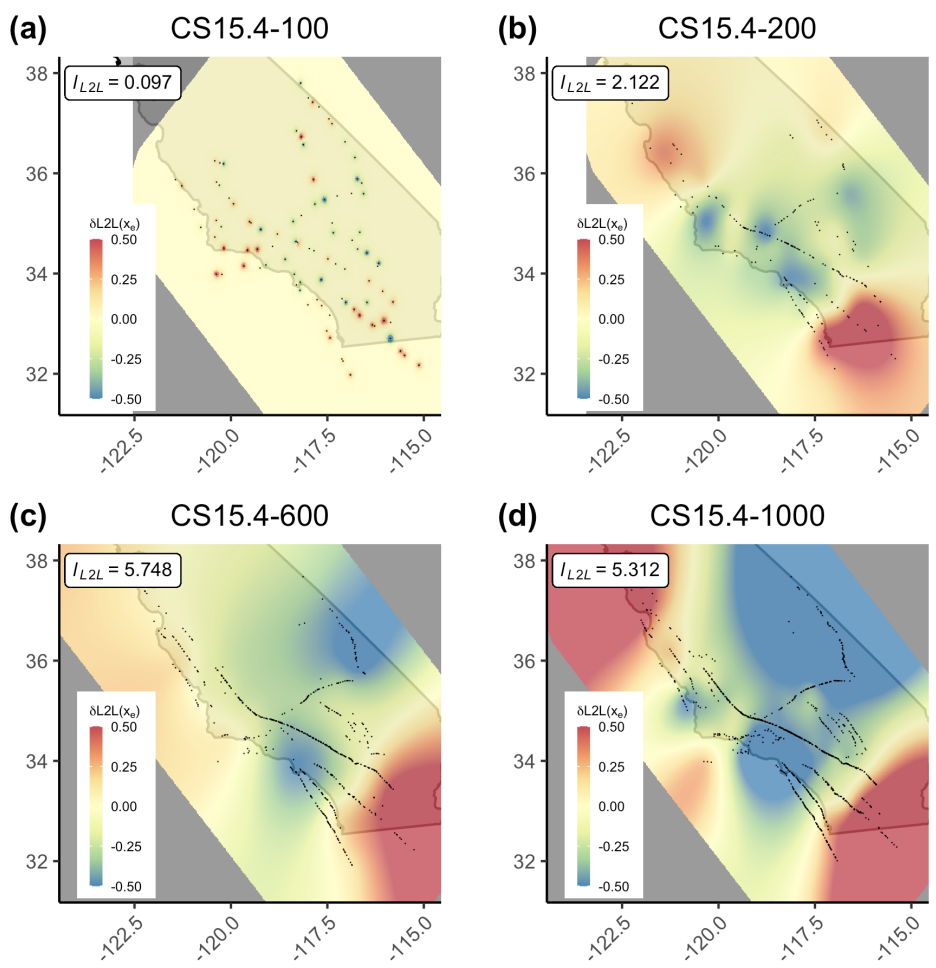

Figure 4. Results of $\delta L 2 L(\vec{x})$ for the four reduced datasets.
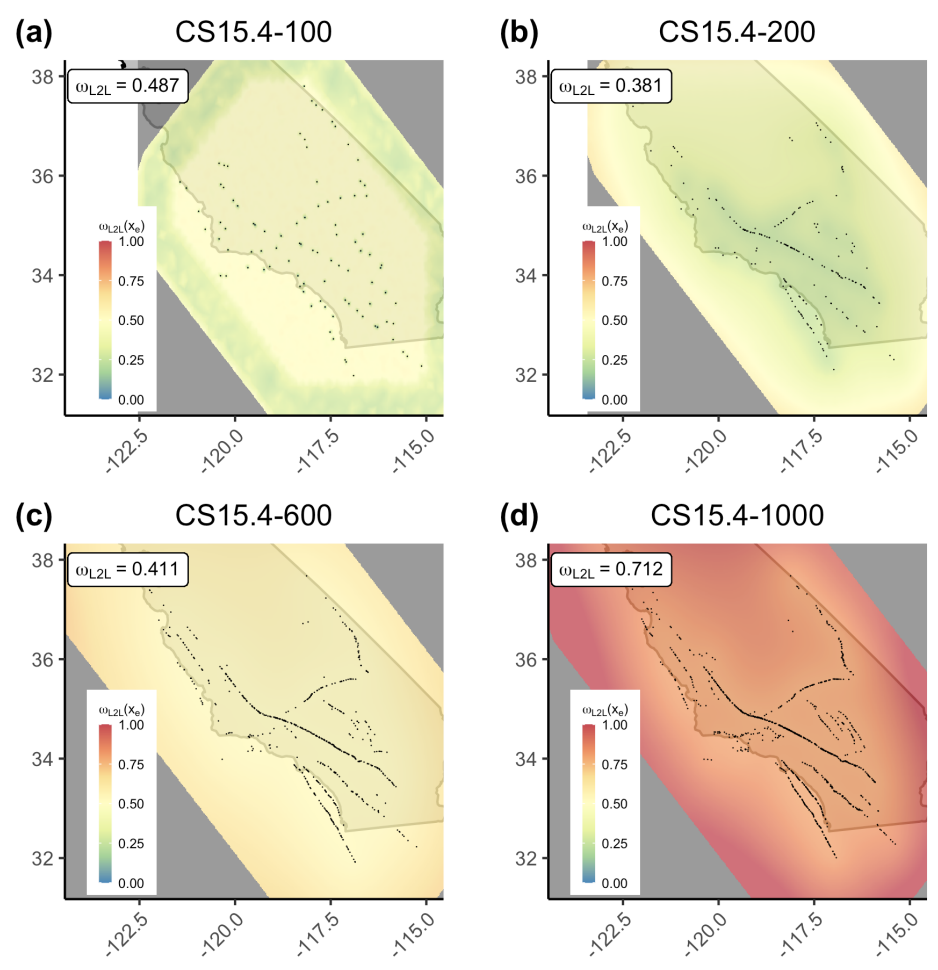
Figure 5. Results of $\omega_{L 2 L}(\vec{x})$ for the four reduced datasets.
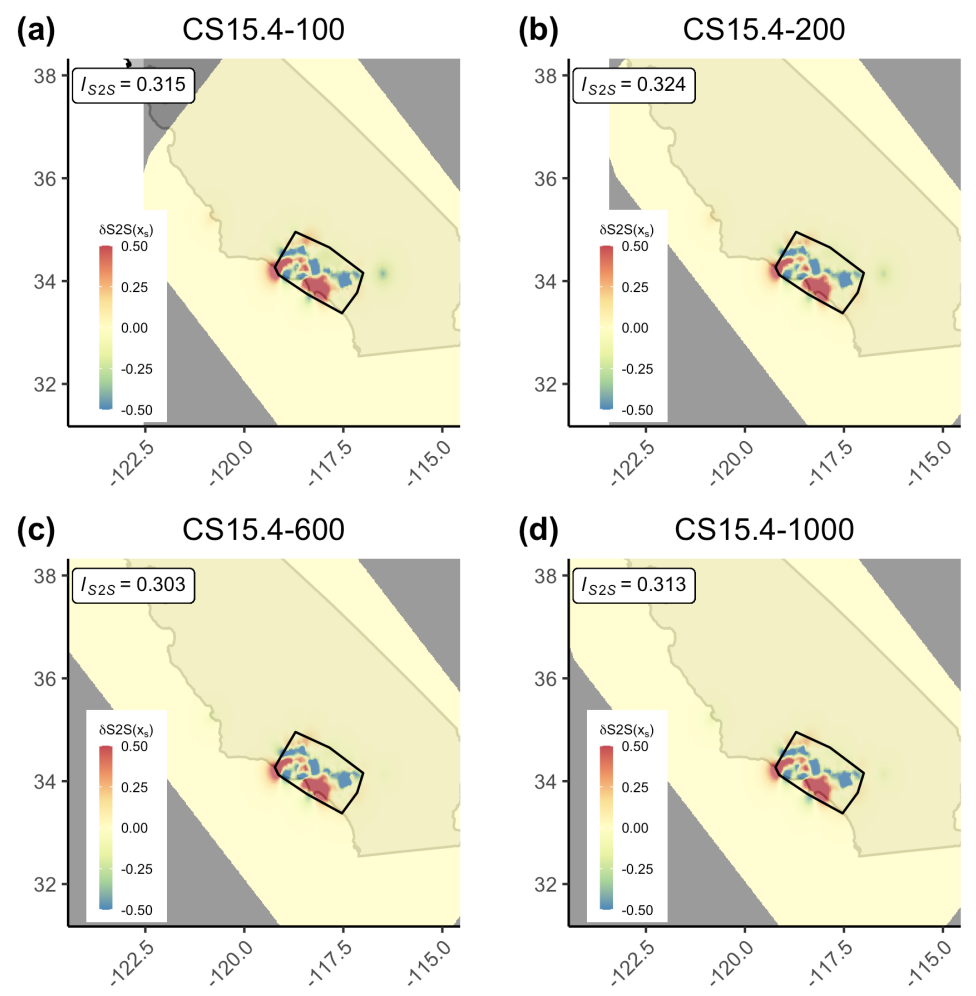

Figure 6. Results of $\delta S 2 S\left(\overrightarrow{x_{s}}\right)$ for the four reduced datasets. The black line denotes the outline of sites.
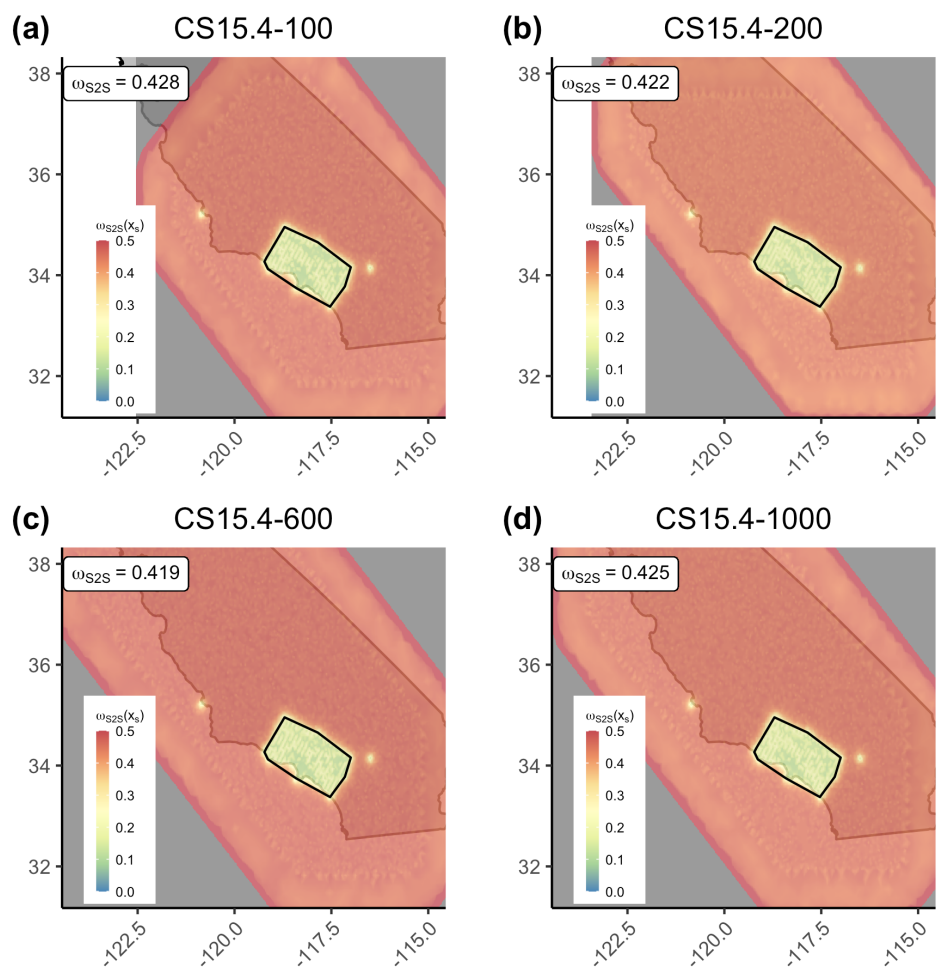
Figure 7. Results of $\omega_{S 2 S}(\vec{x})$ for the four reduced datasets. The black line denotes the outline of sites.
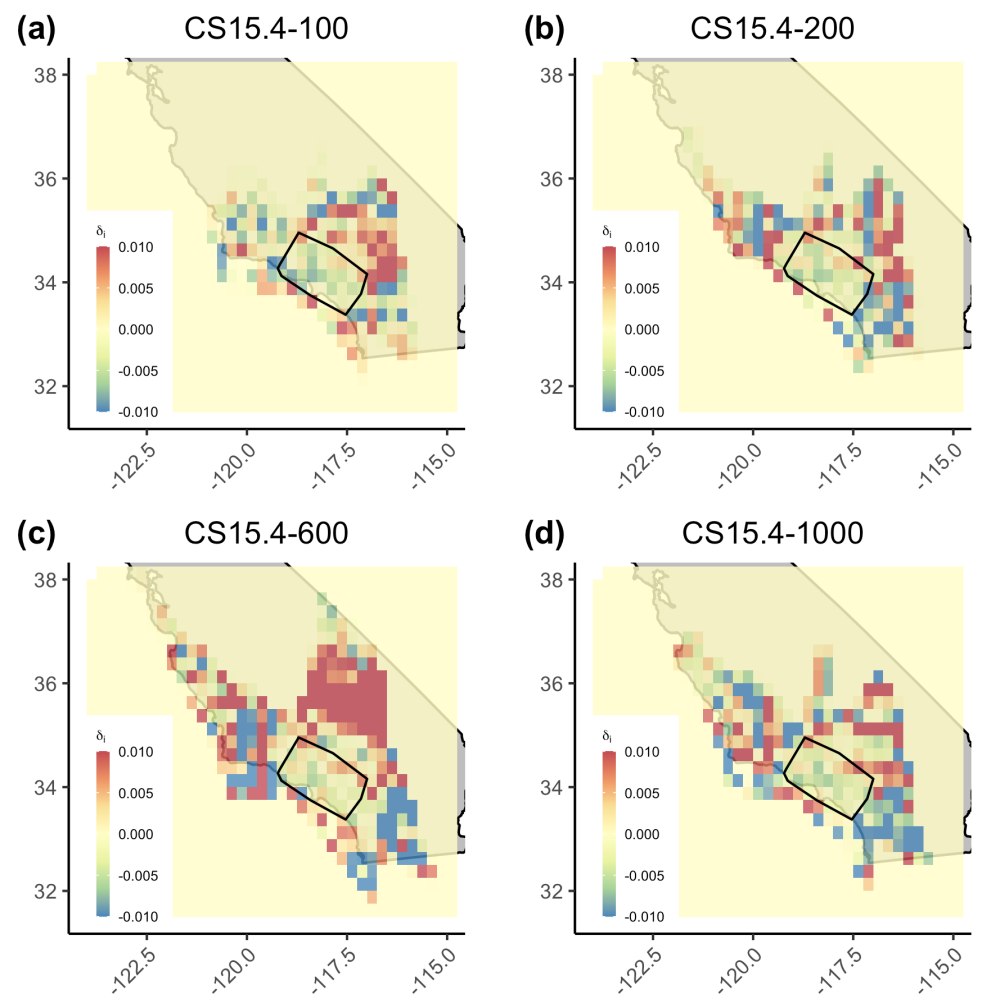

Figure 8. Results of $\delta$ with the 2D cell approach. Black box denotes site coverage. 

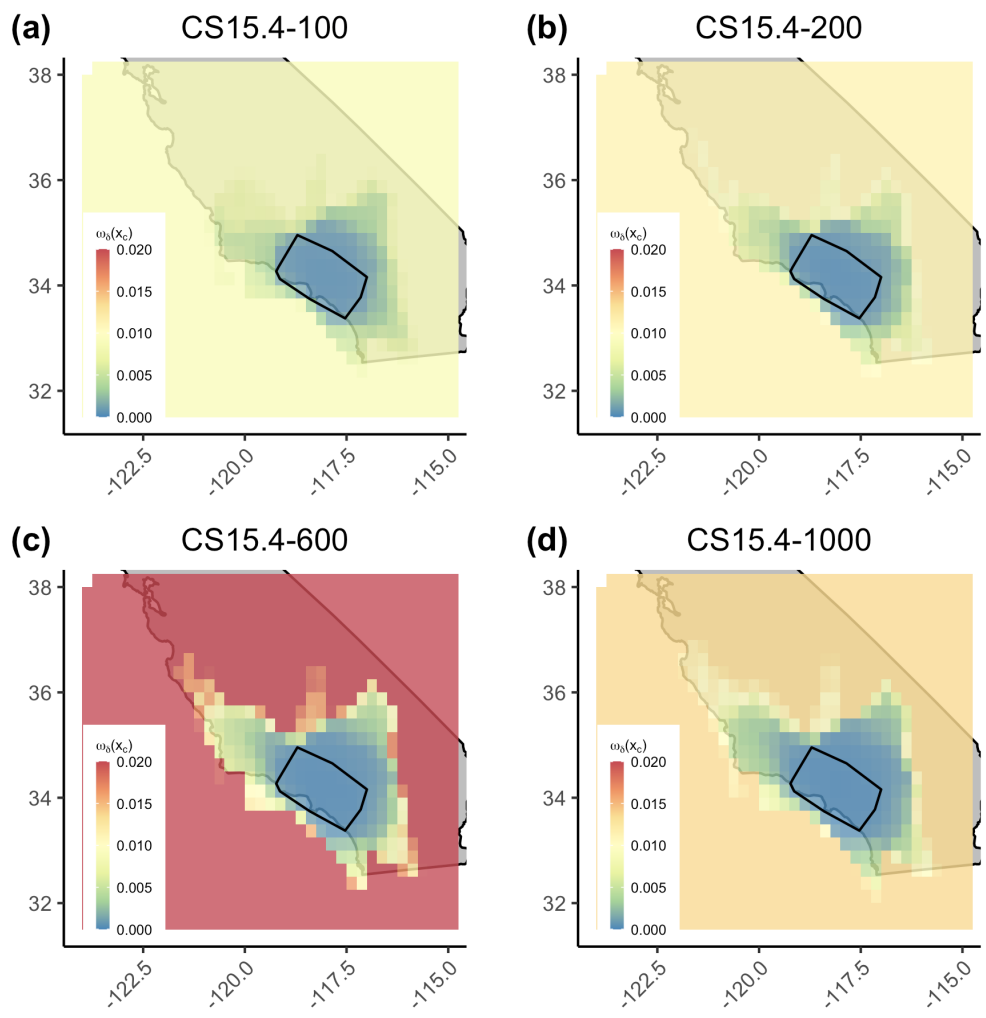

Figure 9. Results of $\omega_{\delta}(\vec{x})$ with the 2D cell approach. Black box denotes site coverage. 

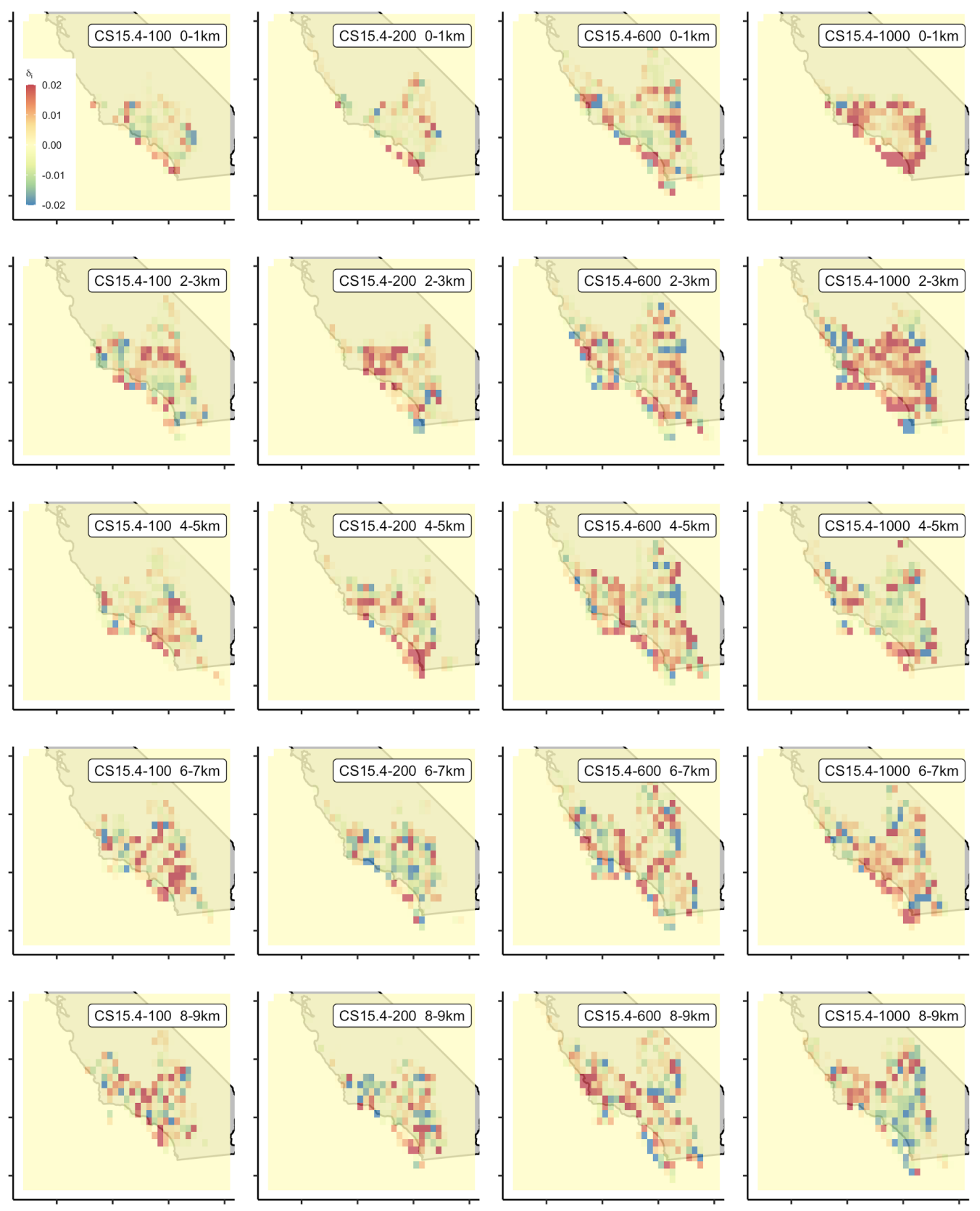

Figure 10. Results of $\delta$ with the 3D cell approach at selected depths. Each row denotes one reduced dataset. 


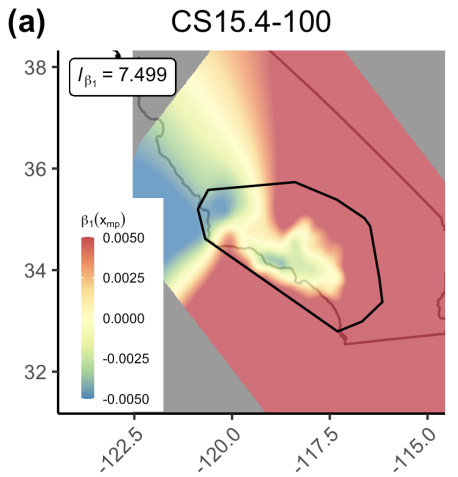

(c)

CS15.4-600

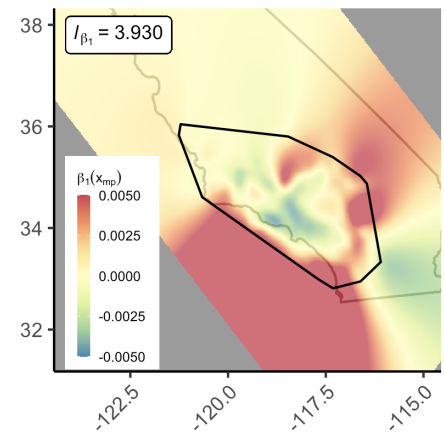

(b) $\quad$ CS15.4-200

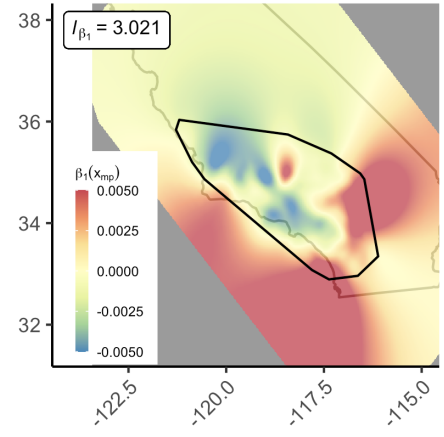

(d) $\quad$ CS15.4-1000

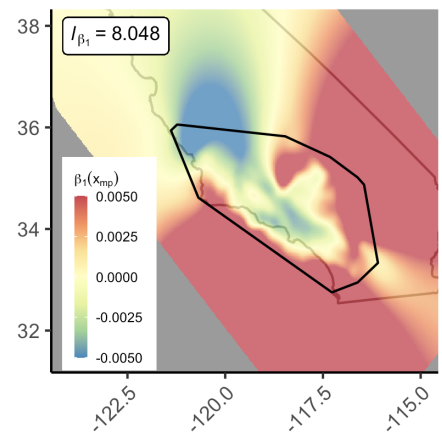

Figure 11. Results of $\beta_{1}\left(x_{m p}\right)$ for the four reduced datasets. The black line denotes the outline of all midpoints.

(a)

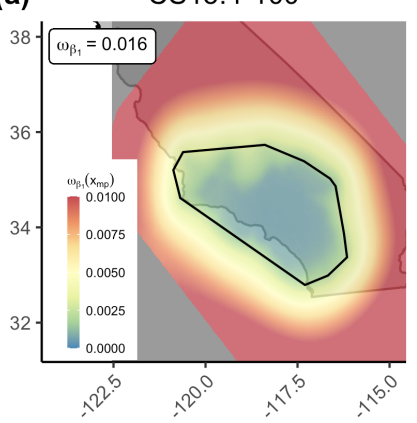

(c)

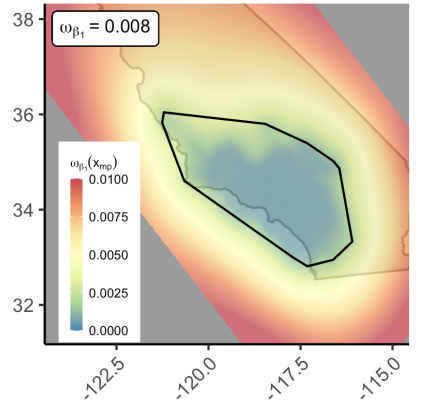

(b) $\quad$ CS15.4-200

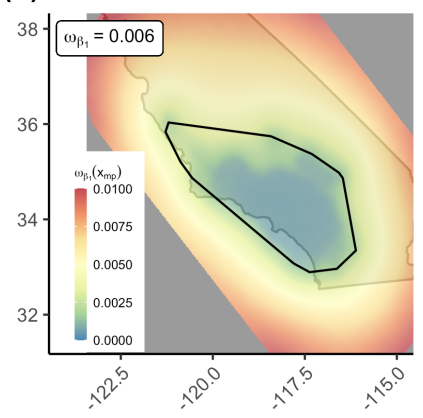

(d)

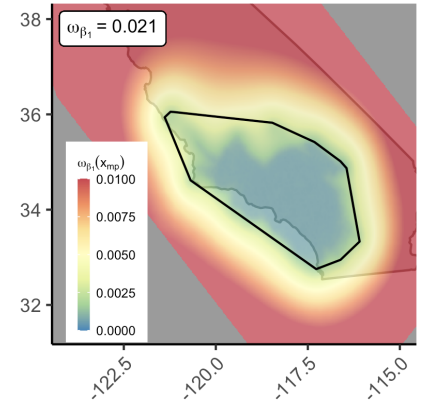


Figure 12. Results of $\omega_{\beta_{1}}\left(x_{m p}\right)$ for the four reduced datasets. The black line denotes the outline of all midpoints.
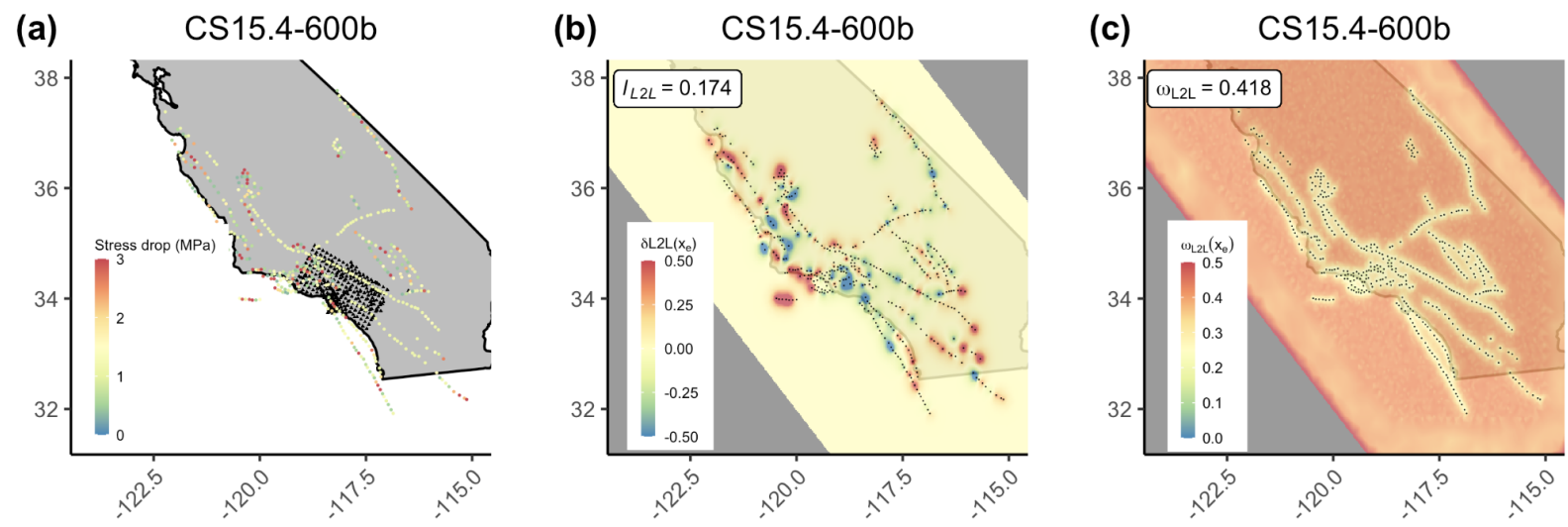

Figure 13. (a) Map of CS15.4-600b. Events are color-coded by stress drop; sites are in black. (b) and (c) Results of $\delta L 2 L(\vec{x})$ and $\omega_{L 2 L}(\vec{x})$ for CS15.4-600b, respectively.
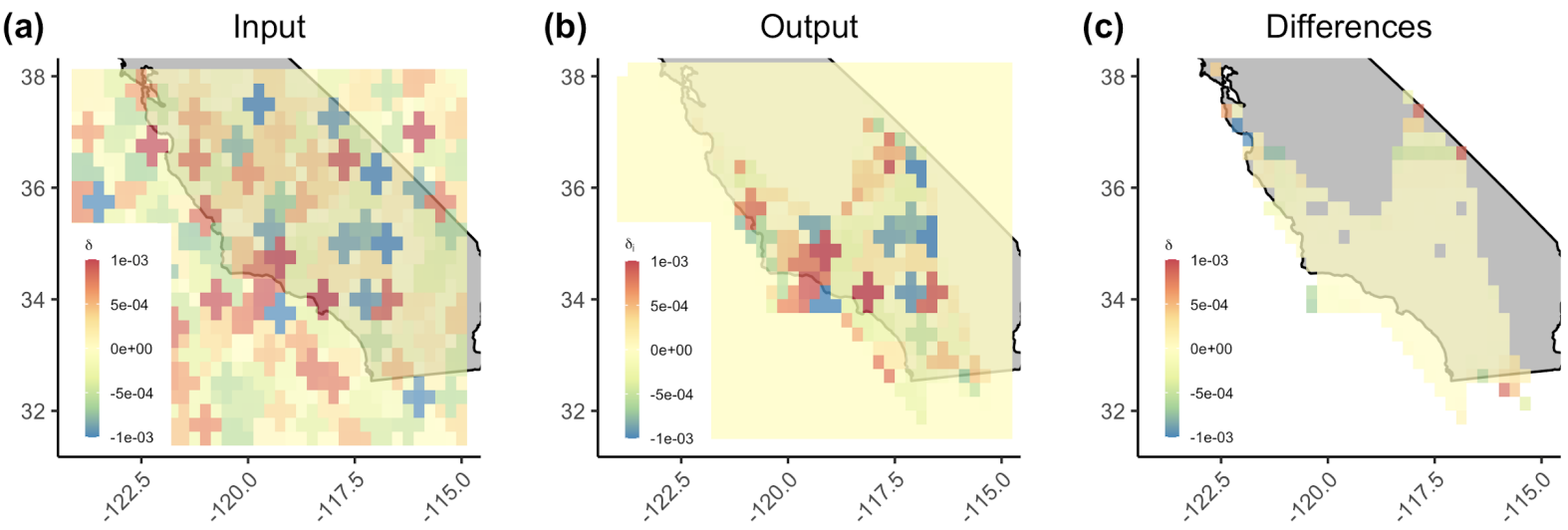

Figure 14. Maps of input $\delta$, output $\delta$ and their differences for the 2D controlled dataset with the 2D cell approach. 

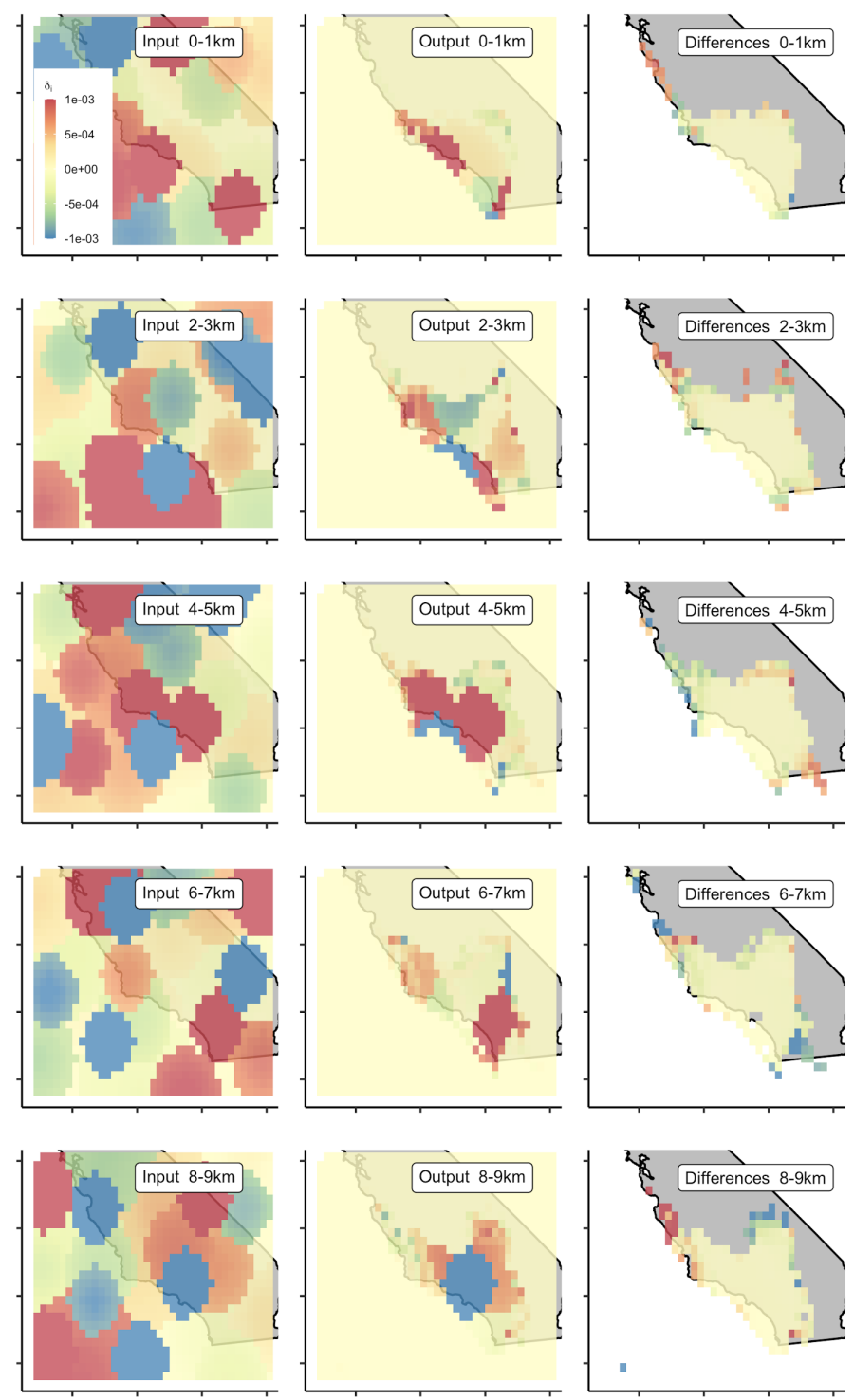

Figure 15. Maps of input $\delta$, output $\delta$ and their differences for the 3D controlled dataset with the $3 \mathrm{D}$ cell approach at selected depths. 

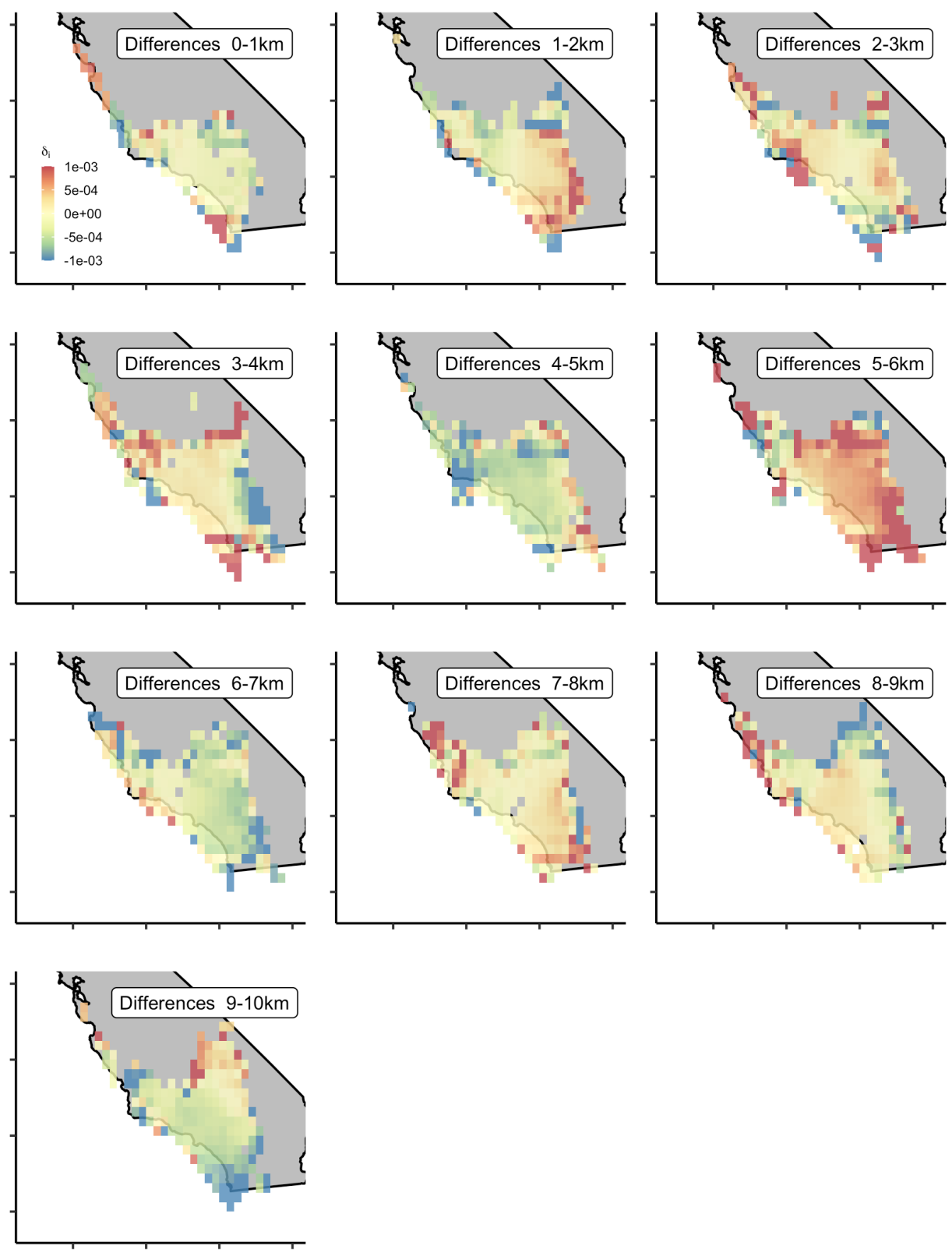

Figure 16. Maps of differences between input and output $\delta$ for the 3D controlled dataset with the $3 \mathrm{D}$ cell approach and shifted hypocenters. 

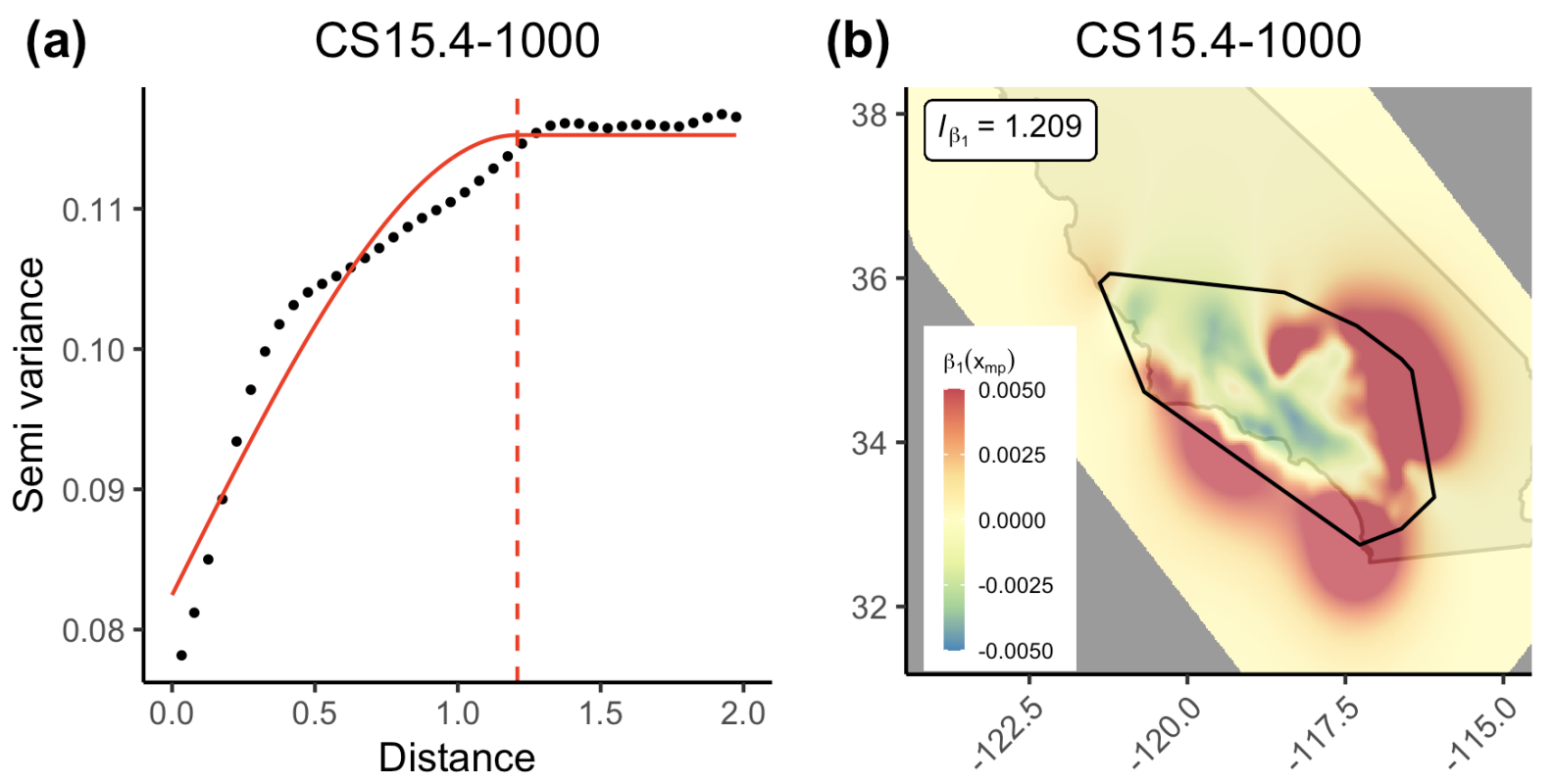

Figure 17. (a) Semivariogram model of path terms computed by mixed-effects regression for CS15.4-1000. The red line denotes the best-fitting spherical model. The red dashed line denotes the range value 1.209 of the best-fitting model. (b) Results of $\beta_{1}\left(x_{m p}\right)$ for CS15.4-1000 with correlation length fixed at 1.209. 

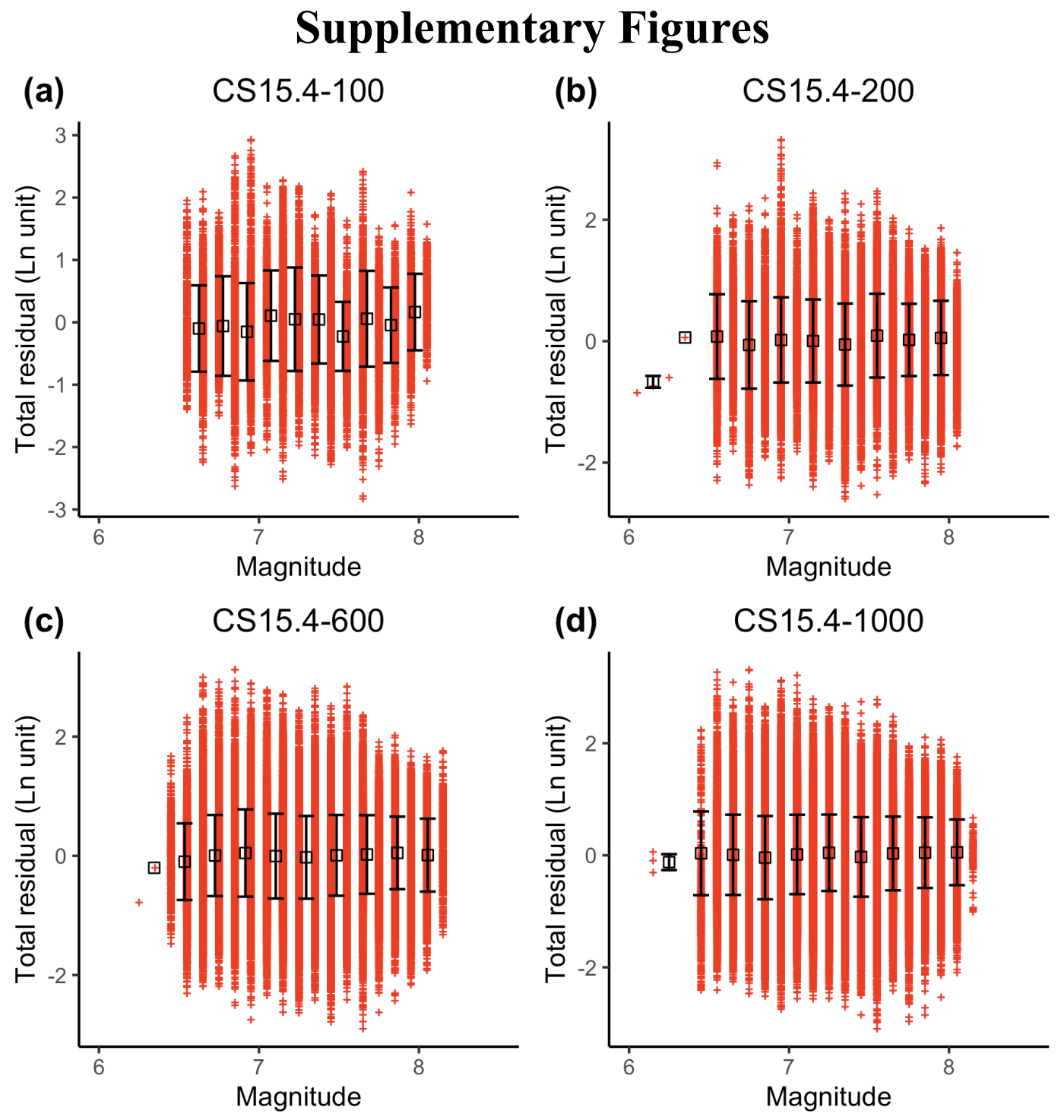

Figure S1. Total residuals from the linear regression versus magnitude for four reduced datasets. The red crosses denote all data points. The black symbols denote the median value and standard deviation of the total residuals. 
(a)

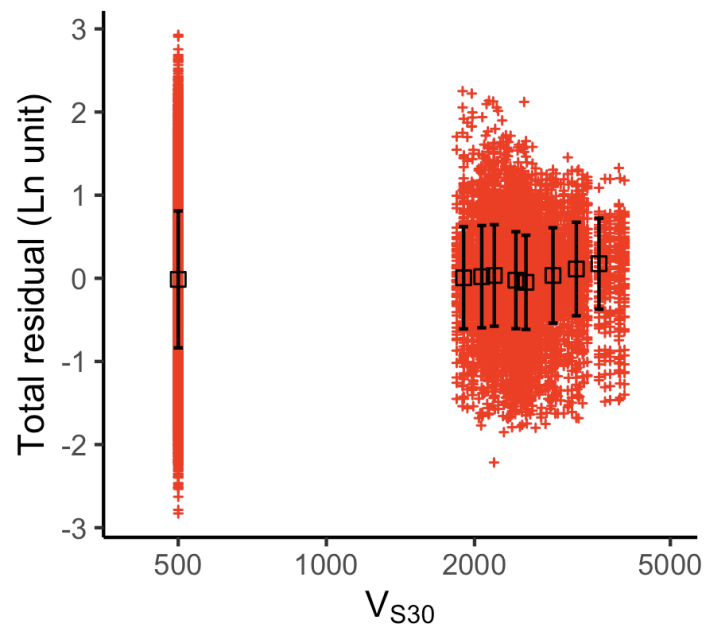

(c)

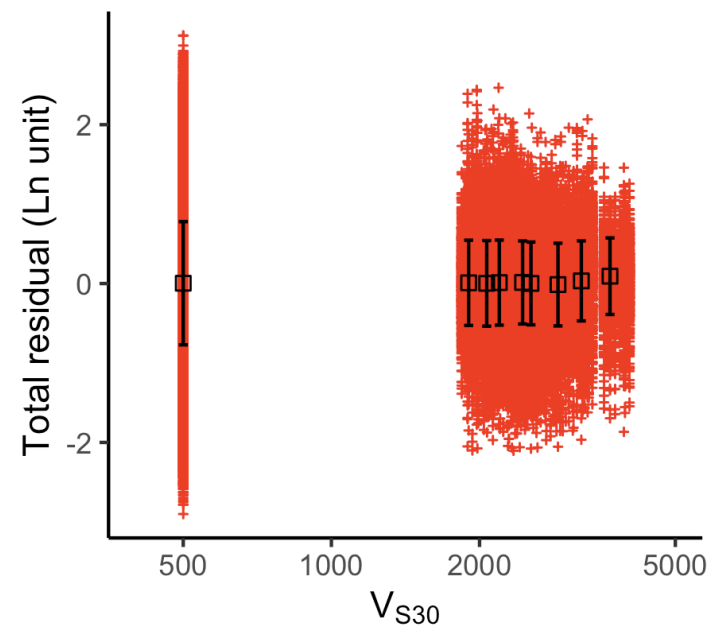

(b) $\quad$ CS15.4-200

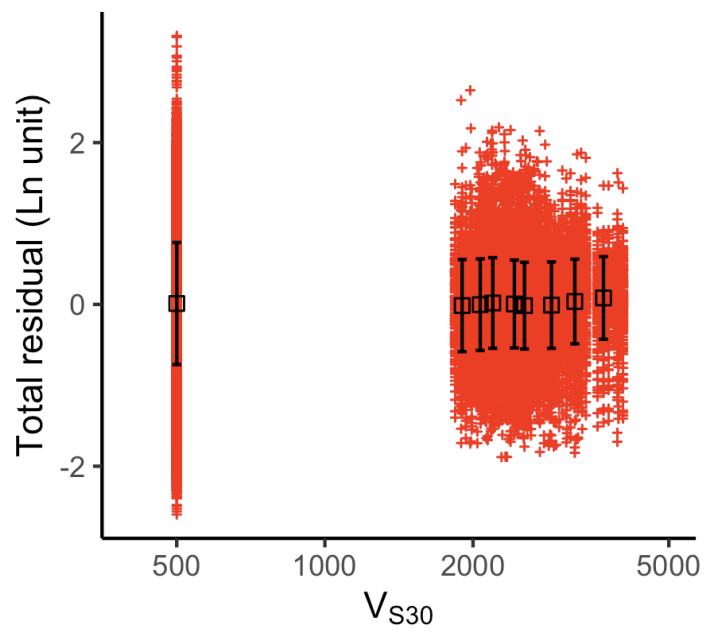

(d) $\quad \operatorname{CS} 15.4-1000$

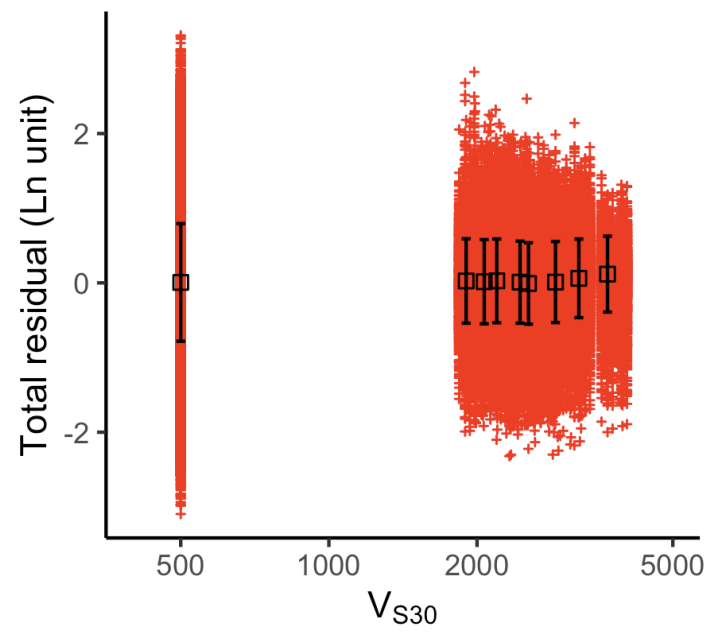

Figure S2. Total residuals from the linear regression versus $\mathrm{V}_{\mathrm{S} 30}$ for four reduced datasets. The red crosses denote all data points. The black symbols denote the median value and standard deviation of the total residuals. 
(a)

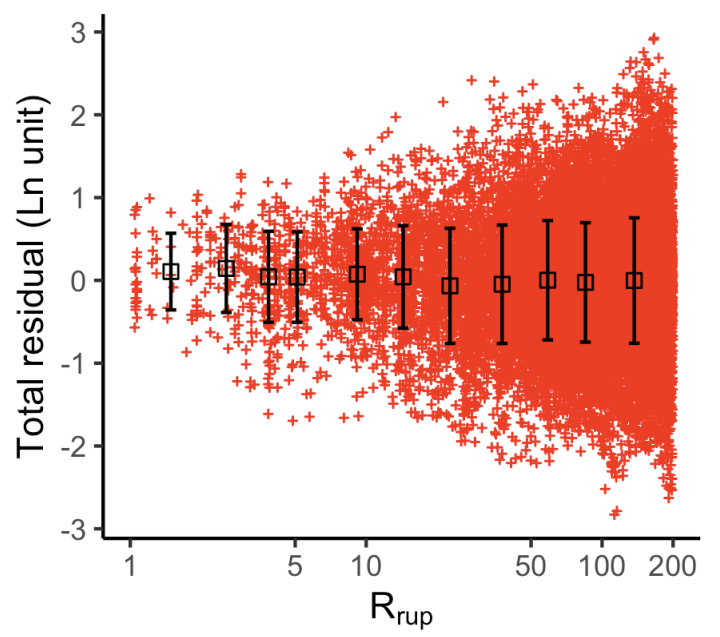

(c)

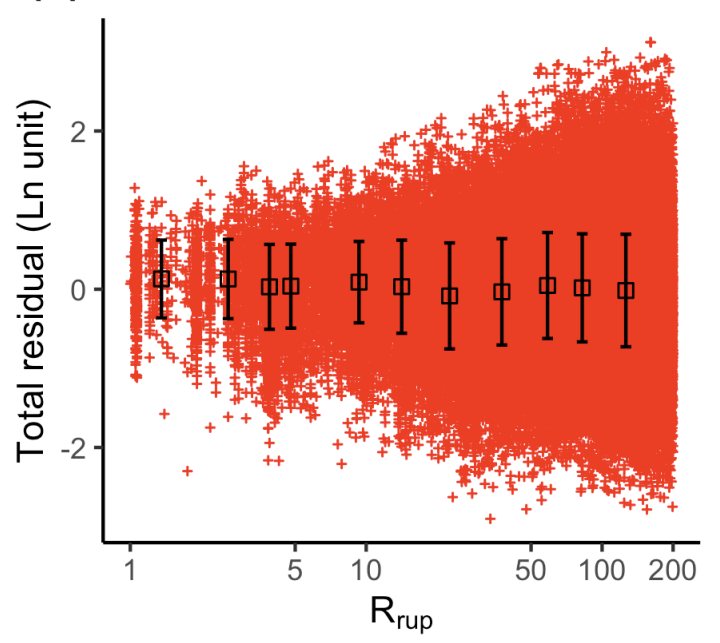

(b) $\quad$ CS15.4-200

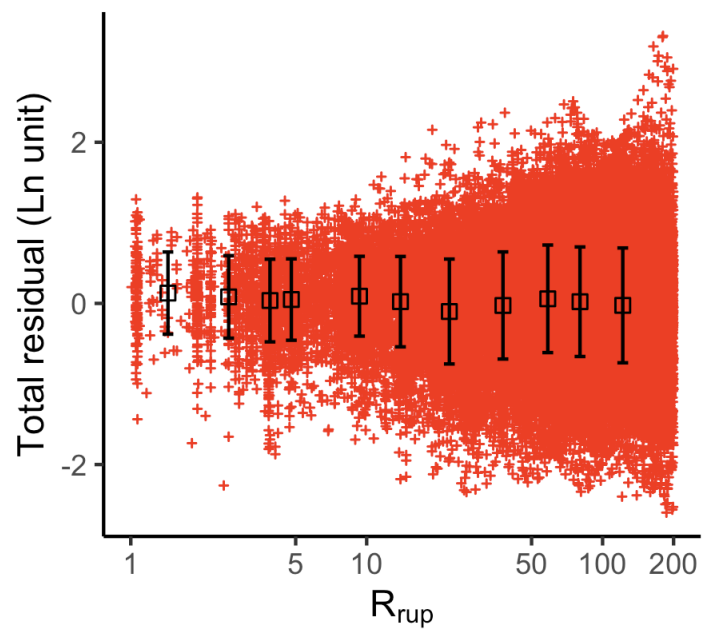

(d) CS15.4-1000

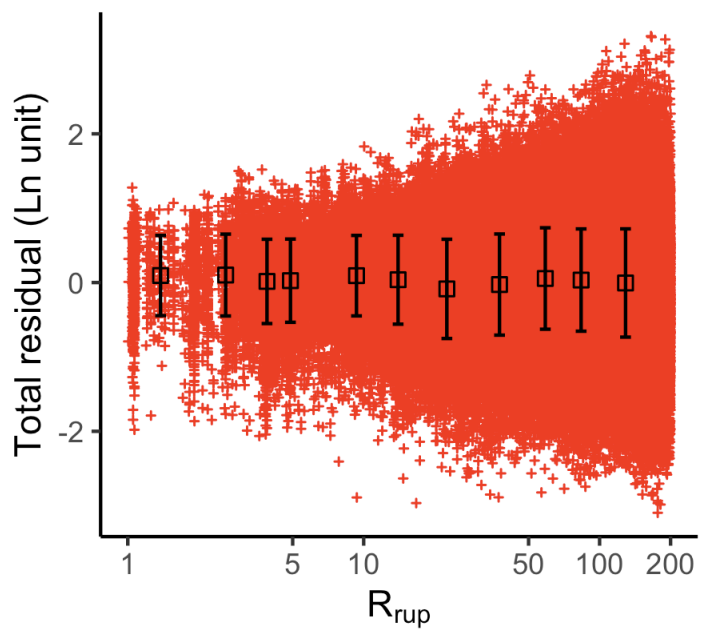

Figure S3. Total residuals from the linear regression versus $\mathrm{R}_{\text {rup }}$ for four reduced datasets. The red crosses denote all data points. The black symbols denote the median value and standard deviation of the total residuals. 

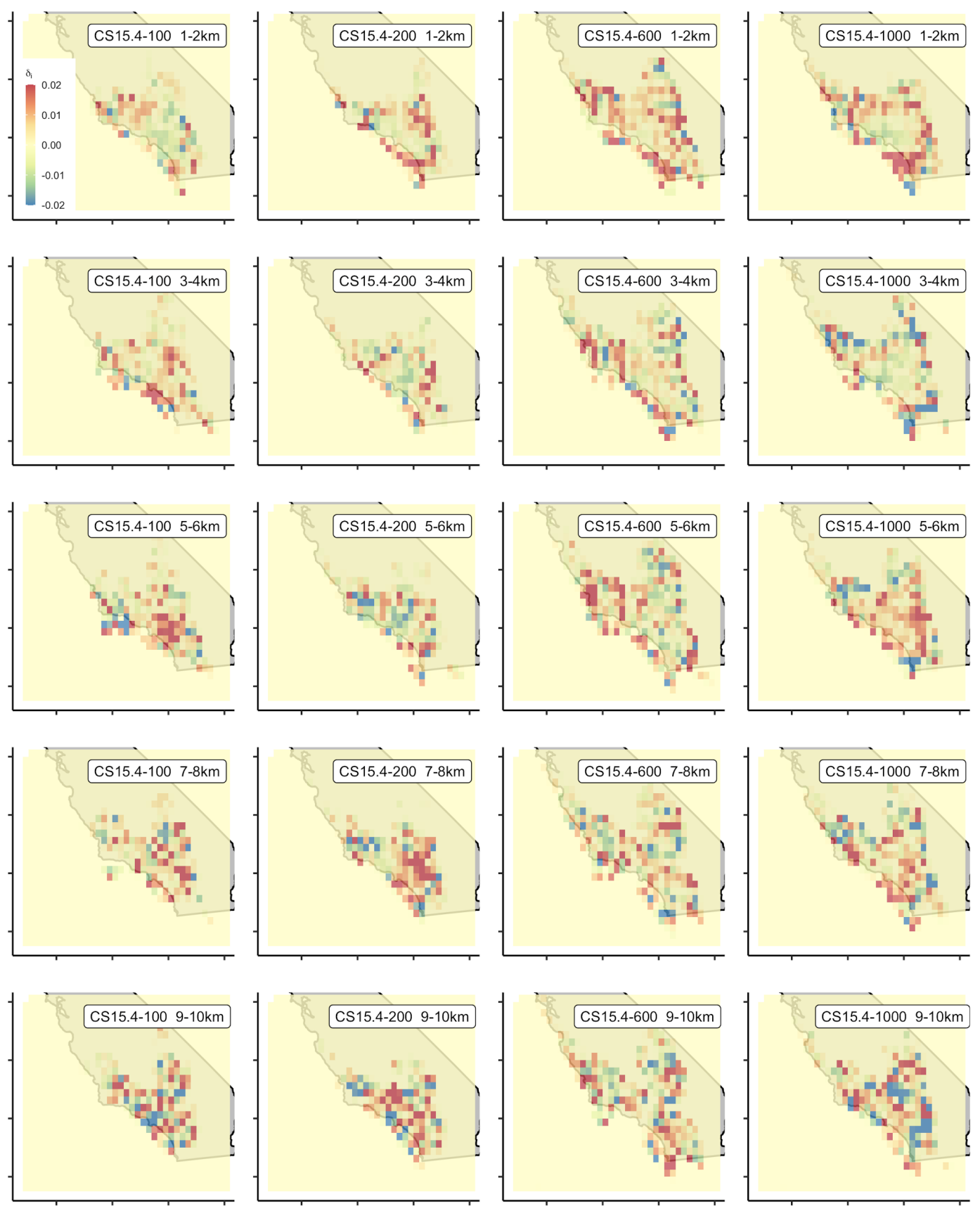

Figure S4. Results of $\delta$ with the 3D cell approach at selected depths. Each row denotes one reduced dataset. 